\title{
Amelioration of hippocampal neuronal morphology and function by mesenchymal stem cell-derived exosomes in APP/PS1 transgenic mice
}

Han Wang

Fourth Military Medical University: Air Force Medical University

Yuqi Liu

Fourth Military Medical University: Air Force Medical University

Junchen Li

Fourth Military Medical University: Air Force Medical University

Tian Wang

Xi'an Jiaotong University

Yue Hei

Fourth Military Medical University: Air Force Medical University

Huiming $\mathrm{Li}$

Fourth Military Medical University: Air Force Medical University

Xue Wang

Xi'an Jiaotong University

Lina Wang

Xi'an Jiaotong University

Ruijing Zhao

Xi'an Jiaotong University

Andrius Baskys

Western University of Health Sciences

Weiping Liu

Fourth Military Medical University: Air Force Medical University

Qianfa Long ( $\square$ lonva@live.cn )

Xi'an Jiaotong University https://orcid.org/0000-0001-8848-9688

\section{Original Article}

Keywords: Exosomes, Alzheimer's disease, neuronal morphology, calcium signaling, action potential, $A \beta$, Nrf2

Posted Date: February 4th, 2021 
DOl: https://doi.org/10.21203/rs.3.rs-162676/v1

License: (c) (1) This work is licensed under a Creative Commons Attribution 4.0 International License. Read Full License 


\section{Abstract}

Mesenchymal stem cell-derived exosomes (MSC-EXO), as a therapeutic agent, have shown great promise in the treatment of neurological diseases. To date, the therapeutic effects and underlying mechanism(s) of MSC-EXO in Alzheimer's disease (AD) are not well understood. The aim of this study was to investigate the action of MSC-EXO on hippocampal neuronal structure and function in APPswe/PS1dE9 (APP/PS1) transgenic mice. Here, the APP/PS1 transgenic mice received a single-dose of MSC-EXO via a tail vein injection, and were then assessed for pathological changes, neuronal morphology alterations, electrophysiological variations, and behavioral deficits. Additionally, the nuclear factor E2-related factor 2 (Nrf2, a key mediator of oxidative injury) signaling pathway was probed by Western blotting in $\mathrm{H}_{2} \mathrm{O}_{2}$ stimulated hippocampal neurons and a mouse model of AD. Our results showed that MSC-EXO therapy inhibited $\beta$-amyloid protein $(A \beta)$ aggregation by reducing protein expression of $6 \mathrm{E} 10$ (marker of deposited amyloid plaques), repaired the synapses and dendritic spines of hippocampal neurons, restored action potentials in hippocampal pyramidal cells, improved cognitive abilities, and reduced memory impairments in a mouse model of AD. Additionally, we found that the Nrf2 signaling pathway participated in the actions of MSC-EXO, both in vitro and in vivo. Together, these data indicate that MSC-derived exosomes ameliorate the deficits in hippocampal neuronal structure and function associated with the Nrf2 signaling pathway in APP/PS1 transgenic mice, suggesting the MSC-EXO as a functional therapeutic agent in AD.

\section{Introduction}

Alzheimer's disease (AD) is a neurodegenerative disorder characterized by a progressive decline in episodic memory as well as deficits in executive functioning and is recognized by the World Health Organization as a global public health priority [1]. Currently, $60-80 \%$ of the total population suffering from dementia is ascribed to $A D$, and elderly individuals are considered to be more susceptible $[2,3]$. Previous studies have suggested that a cascade of neuropathological alterations occur in Alzheimer's disease including dystrophic neurites, astrogliosis and microglial activation, neuronal and synaptic loss, ion channel dysfunction, and mitochondrial injury. Among which, amyloid plaque deposition and neurofibrillary tangles are believed to be the major pathological changes that occur in the initiation and progression of $A D$ [4-6]. Although multiple treatment strategies, including cell-therapy, have been used against amyloid plaque aggregation and neural dysfunction in AD models and patients [7, 8], immense challenges remain including reconstructing neuronal structure and function.

Undoubtedly, the development of regenerative medicine using stem cell-therapy is useful for neurodegenerative disorders $[9,10]$. Specifically, mesenchymal stem cells (MSCs) show great promise in the treatment of $A D$ owing to their biological characteristics, including low immunogenicity, antiinflammatory properties, the ability to expand in culture, and the ease of isolation [11]. However, accumulating evidence suggests that the paracrine action of MSCs plays a crucial role in neuroprotection and immunomodulation of brain diseases $[12,13]$. Moreover, the exosomes (EXO) secreted by MSCs are more effective as a restorative therapy in neurological injury than their parental cells [14]. Notably, EXO 
contain a large number of therapeutic agents (e.g., RNA, protein, DNA, etc.) as well as have the ability to permeate the blood-brain barrier and are easy to use and preserve, making them favorable for development in basic and preclinical research [15]. Recent studies indicate that the MSC-secretome as well as exosomes show positive effects on oxidative stress, neuroinflammation, $\beta$-amyloid protein (A $\beta$ ) aggregation, and the associated memory impairments in APPswe/PS1dE9 (APP/PS1) transgenic mice (a widely used AD model) $[16,17]$. However, the action of MSC-EXO in neuronal dysfunction is not well understood.

Previous studies, including our own, have shown that MSC-EXO can target neurons and promote neuroregeneration following brain injury [18], suggesting that exosome-therapy exhibits direct neuroprotective effects in neurological diseases in addition to its immunomodulatory effects. Interneuron dysfunction, including altered synaptic plasticity, impaired excitability, and intracellular calcium $\left(\mathrm{Ca}^{2+}\right)$ disruption have emerged as potential mechanisms underlying the cognitive deficits in $\operatorname{AD}[19,20]$. Additionally, oxidative damage is a key component in the pathogenesis of $A D[21,22]$. Thus, modulating these mechanisms may help to improve brain function in these conditions.

In this study, we aimed to develop MSC-EXO as a cell-free nanotherapeutic to restore hippocampal neuronal structure and function in APP/PS1 transgenic mice. We found that exosome-therapy repaired dendritic spine morphology, action potentials, and $\mathrm{Ca}^{2+}$ transients of hippocampal neurons, as well as ameliorated $A \beta$ aggregation and the cognitive deficits in a mouse model of $A D$. We also found that the nuclear factor E2-related factor 2 (Nrf2) signaling pathway participated in the oxidative defense produced by MSC-EXO, both in vitro and in vivo. Collectively, our results demonstrate the novel therapeutic effects of MSC-EXO in AD and shed light on the mechanism underlying its efficacy.

\section{Materials And Methods}

\section{Animals}

APPswe/PS1dE9 (APP/PS1) transgenic mice (male, SPF grade) and age-matched wild type (WT) littermates were purchased from Beijing HFK Bioscience Co., LTD. The APP/PS1 mice over-express the delta exon 9 variant of presenilin 1 (PS1) and the Swedish mutation of $\beta$-amyloid precursor (APP). For primary neuron culture, pregnant (embryo at 15-18-day) C57B/6 mice were obtained from the Experimental Animal Center of the Fourth Military Medical University. Animals were housed in a controlled environment under conventional conditions with a $12 \mathrm{~h}$ light / dark cycle and food and water provided ad libitum. All procedures, including the behavioral test, were performed during the light cycle.

\section{MSCs culture and flow cytometry (FCM) identification}

Human umbilical cord tissues were harvested from fetuses with natural labor and the donors were selected from full-term puerpera in good health. Informed consent was obtained from all the donors. The procedures were approved by the Guideline of the Ethical Committee of the Xi'an Central Hospital (No. 20180715-1), in accordance with the guidelines of the National Institutes of Health, USA. 
MSCs were obtained via enzymatic digestion of Wharton's jelly within the umbilical cord and expanded using standard medium, a-minimum essential medium (a-MEM; Gibco, New York, USA) supplemented with $10 \%$ fetal bovine serum (FBS; Gibco, New York, USA), $100 \mathrm{U} / \mathrm{mL}$ penicillin and $100 \mu \mathrm{g} / \mathrm{mL}$ streptomycin (Gibco, New York, USA) as previously described [23].

The well-grown 3rd passage MSCs were washed twice with precooled phosphate-buffered saline (PBS). The cells were then separated into Eppendorf (EP) tube at $5 \times 10^{5} / 1.5 \mathrm{~mL}$. The cells were resuspended with $100 \mu \mathrm{L}$ of PBS and then the following antibodies were added: Anti-human CD29 (Biolegend, 303003, San Diego, CA, USA), CD44 (Biolegend, 338807, San Diego, CA, USA), CD105 (Biolegend, 800503, San Diego, CA, USA) in the PE channel; Anti-human CD34 (Biolegend, 343503, San Diego, CA, USA), CD45 (Biolegend, 304005, San Diego, CA, USA) and CD90 (Biolegend, San Diego, CA, 328107, USA) in the FITC channel. Luciferin-labeled homozygous control antibodies were added to the homozygous pair tube as a control. The cells were incubated in dark for $30 \mathrm{~min}$ at $4{ }^{\circ} \mathrm{C}$. The cells were washed three times with a PBS solution and then loaded into the detection tube for flow cytometry detection.

\section{MSC-EXO isolation and characterization}

MSCs (3rd-5th passage) were washed twice with PBS upon coming to 70\% confluency, and then replaced with medium containing exosome-depleted FBS. The exosome-depleted FBS was prepared by 18 h ultracentrifugation of FBS at 100,000g using an SW32Ti rotor (XPN-100, Beckman, California, USA). After $24-48 \mathrm{~h}$, the supernatants were harvested and stored at $-80^{\circ} \mathrm{C}$ until processing.

Ultracentrifugation was employed to concentrate the EXO released from the MSCs as previously described [24]. Briefly, fresh or frozen cell culture supernatants were centrifuged at $300 \mathrm{~g}$ for $10 \mathrm{~min}$ to remove cellular debris (TX-400 rotor, ST16R, Thermo Fisher, Massachusetts, USA), followed by 2,000g for $20 \mathrm{~min}$ to remove smaller cellular debris. Subsequently, supernatants were centrifuged at $10,000 \mathrm{~g}$ for 30 min to remove larger vesicles and apoptotic bodies (F15-6x100y rotor, ST16R, Thermo Fisher, Massachusetts, USA). EXO were then pelleted by ultracentrifugation at $100,000 \mathrm{~g}$ for $70 \mathrm{~min}$ in a swing rotor (SW32Ti, XPN-100, Beckman, California, USA). The pellet was washed with ice-cold PBS, resuspended in PBS and stored at $-80^{\circ} \mathrm{C}$.

EXO quantification was performed by determination of the protein contents using a bicinchoninic acid assay (BCA; Beyotime, P0010, Beijing, China). In order to assess the size distribution and morphology of the EXO, the vesicles were diluted in sterile PBS and subjected to a nanoparticle tracking analysis (NTA; Nanosight LM10, Malvern, Worchestershire, UK) and transmission electron microscopy (TEM; JEM-1400, JEOL Ltd., Japan). Western blotting was used to assay the representative markers of EXO including CD63 (1:200, Santa Cruz, USA), CD9 (1:1000, Abcam, California, USA), and TSG101 (1:5000, Abcam, California, USA).

\section{Labeling of MSC-Exo with Alexa 594}


A MSC-Exo suspension (1-2 mg/ml) was prepared in advance. As previously described [25], C5 Maleimide-Alexa 594 (CM-A954; Invitrogen, A10256, California, USA) was added to $50 \mu \mathrm{L}$ of the MSC-Exo for $1 \mathrm{~h}$ at room temperature (RT) in the dark, and at the same time, ECO spin columns (MW3000, Invitrogen) were prepared in accordance with the manufacturer's instructions. During this incubation, powdered resin was hydrated for $20 \mathrm{~min}$ at R/T. Spin columns were then placed in the collection tubes and an AllegraX-15R (Beckman, USA) was used to centrifuge them for 2 min (750g). The collection tubes were discarded and then the labelled MSC-Exo were added to the resin. Columns were then placed in 1.5 $\mathrm{ml}$ tubes and centrifuged for $3 \mathrm{~min}(750 \mathrm{~g})$ to collect the labeled MSC-Exo. Isopycnic PBS was performed in parallel to all the protocols as controls. Subsequently, the labelled MSC-Exo was stored at $-80^{\circ} \mathrm{C}$. Labelled MSC-Exo were diluted to the appropriate concentration according to the experimental requirements and filtered through a $0.22 \mu \mathrm{m}$ filter to remove dye aggregates before use.

\section{SH-SY5Y cell lines and primary hippocampal neuron cultures}

Confocal dishes or 96 -well plates were coated with poly (I-lysine) for $4 \mathrm{~h}$ in a cell incubator $\left(37^{\circ} \mathrm{C}\right)$, washed by sterile water three times, and subsequently air-dried using a clean bench. Hippocampal tissue was stripped from 16-18-day old embryos of mice in a sterile environment and digested at $37^{\circ} \mathrm{C}$ with $0.125 \%$ trypsin for 10 min and then stopped by Dulbecco's modified Eagle's medium (DMEM) containing $10 \%$ FBS. The number and concentration of viable cells were determined by trypan blue exclusion using an automated cell counter (JSY-SC-031, BodBoge, China). Whereafter, the cells were plated in neuronal growth and maintenance medium containing neurobasal medium (Gibco, New York, USA), 2\% B27 (Gibco, New York, USA), $100 \mathrm{U} / \mathrm{ml}$ penicillin-streptomycin, and $2 \mathrm{mM}$ L-glutamine (Gibco, New York, USA). In the follow-up study, the purification of hippocampal neurons reached more than $98 \%$. The culture medium was changed every three days, and primary neurons could be used for subsequent experiments after about $1 \mathrm{w}$.

Two SH-SY5Y cell lines (APPwt, APPswe), expressing wild type A $\beta$ precursor protein (APP) and APP with the Swedish mutation, respectively, were obtained from iCell Bioscience Inc, Shanghai. SH-SY5Y APPwt cells generate min quantities of A $\beta$ peptides while the SH-SY5Y APPswe cells produce high concentrations of $A \beta[26,27]$. The cells were grown in DMEM supplemented with $10 \%$ FBS, $1 \%$ MEM NEAA, $1 \%$ penicillin/streptomycin in $5 \% \mathrm{CO}_{2}$ in air at $37^{\circ} \mathrm{C}$ in a humidified incubator.

\section{A $\beta$ Oligomers preparation}

$A \beta$ Oligomers were prepared as previously described with minor modifications [28]. A $\beta$ 1-42 (AnaSpec, San Jose, CA, USA) was dissolved in 1,1,1,3,3,3-hexafluoro-2-propanol (HFIP, Sigma-Aldrich, 52517, California, USA) to $1 \mathrm{mM}$, which was naturally volatilized in the fume hood at RT for 2-4 h. Then, the clear solution was frozen and drained by the lyophilizer to form an $A \beta$ peptide film, which was stored at $-80^{\circ} \mathrm{C}$ or prepared directly. The peptide was subsequently dissolved in Dimethyl sulfoxide (DMSO; SigmaAldrich, D2650, California, USA) to $5 \mathrm{mM}$, followed by bath sonication for $10 \mathrm{~min}$, and then diluted to 100 $\mathrm{M}$ with neurobasal medium and incubated at $4^{\circ} \mathrm{C}$ for $24 \mathrm{~h}$. The insoluble $A \beta$ was removed by 
centrifugation at $10000 \mathrm{~g}$ for $5 \mathrm{~min}$. For in vitro applications, we used $10 \mathrm{mM} \mathrm{A} \beta$ as our treatment condition based on previous studies [29].

\section{MSC-EXO administration and tracking}

MSC-Exo were prepared at a concentration of $1 \mathrm{mg} / \mathrm{ml}$ in sterile PBS and stored at $-80^{\circ} \mathrm{C}$. For in vitro experiments, $10 \mu \mathrm{g} / \mathrm{ml} \mathrm{MSC-Exo} \mathrm{were} \mathrm{added} \mathrm{to} \mathrm{the} \mathrm{culture} \mathrm{medium} \mathrm{of} \mathrm{the} \mathrm{primary} \mathrm{neurons} \mathrm{and} \mathrm{SHSY5Y}$ APPswe and incubated for $12 \mathrm{~h}$ to ensure incorporation of the exosomes. Neurons were subsequently exposed to $10 \mathrm{mM} \mathrm{A} \beta$ (A $\beta+E X O$ group) for $24 \mathrm{~h}$. Neurons in the Control and $A \beta+P B S$ groups were treated with PBS and $10 \mathrm{mM} A \beta$. For in vivo studies, nine-month-old APP/PS1 mice were randomly divided into the $A D+$ saline and $A D+M S C$-Exo groups and each mouse were injected with $150 \mu \mathrm{L}$ of saline or MSCEXO $(50 \mu \mathrm{g} / 150 \mu \mathrm{L})$ into the caudal vein, respectively. Age-matched WT mice received an equivalent volume of saline as the naive group. Also, CM-A594-labelled MSC-EXO were used as the treatment in the animal $(n=3)$ models. Then, the tissue samples were fixed with $4 \%$ paraformaldehyde and stained with NeuN to observe the labeled MSC-EXO in vivo.

\section{Calcium imaging}

Intracellular $\mathrm{Ca}^{2+}$ oscillations in primary hippocampal neurons (control, $\mathrm{A} \beta+\mathrm{PBS}$, and $\mathrm{A} \beta+\mathrm{EXO}$ group; $\mathrm{n}$ = 5 per group) were detected using the calcium indicator Fluo-8 AM (Abcam, AB142773, California, USA) as previously described [30,31]. To load the dye into the hippocampal neurons, the primary cultures were rinsed in phenol red-free DMEM containing $4 \mu \mathrm{M}$ Fluo-8 AM and 0.08\% Pluronic F127 (Life Technologies, CA, USA) for 20 min at $37^{\circ} \mathrm{C}$. Subsequently, the cells were washed three times with Hank's balanced salt solution (HBSS) and the samples were stored in artificial cerebrospinal fluid (ACSF) containing $124 \mathrm{mM}$ $\mathrm{NaCl}, 25 \mathrm{mM} \mathrm{NaHCO}$, $2.5 \mathrm{mM} \mathrm{KCl}, 1 \mathrm{mM} \mathrm{KH} 2 \mathrm{PO} 4,2 \mathrm{mM} \mathrm{CaCl}$, $2 \mathrm{mM} \mathrm{MgSO}$, and $10 \mathrm{mM}$ glucose. Resting state $\mathrm{Ca}^{2+}$ levels were recorded for $20 \mathrm{sec}$, and then $10 \mathrm{mM}$ adenosine monophosphate (ATP) was added into the ACSF to stimulate a $\mathrm{Ca}^{2+}$ influx. The fluorescence images were captured every sec for $70 \mathrm{sec}$ at the wavelength of $488 \mathrm{~nm}$ using a confocal microscope (Olympus, FV3000, Japan). The image analysis software Cellcens (Olympus, Japan) was used to measure calcium influx and resting $\mathrm{Ca}^{2+}$ levels of the individual hippocampal neurons. More than 80 cells from at least three independent experiments were analyzed for each group using Igor Pro software (WaveMetrics in Oregon, USA).

\section{TEM of hippocampus}

The TEM study was conducted as previously described with minor modifications [32]. Tissue $\left(1 * 1 * 2 \mathrm{~m}^{3}\right)$ was quickly harvested in a fixative solution, and the brain was removed and sliced in less than 2 min. Tissue blocks were fixed for $24 \mathrm{~h}$ with $4 \%$ glutaraldehyde, and then washed three times in a $1 \%$ PBS solution, once for $15 \mathrm{~min}$, followed by further fixation with $1 \%$ osmium tetroxide for $2 \mathrm{~h}$. Samples were dehydrated using graded ethanol, permeated with a mixture of acetone and Eponate 812, and embedded in an embedding plate with Eponate 812. The embedded block was trimmed into a trapezoid and mounted onto blank resin stubs for ultrathin sectioning. After being counterstained with uranium dioxane 
acetate and lead citrate, the sections were observed under a TECNAI Spirit electron microscope (Thermo Fisher, USA).

\section{Whole-cell patch-clamp recording}

Experimental animals' brain tissue was cut into $300 \mu \mathrm{m}$ hippocampal coronal slices. An action potential was induced by positive current injection, which brought the membrane potential to $-50 \mathrm{mV}--55 \mathrm{mV}$. The slices were immersed in oxygenated $\left(95 \% \mathrm{O}_{2} / 5 \% \mathrm{CO}_{2}\right)$ ACSF at RT $\left(22-25^{\circ} \mathrm{C}\right)$ until use, and then placed in a recording chamber and perfused with extracellular solution containing $124 \mathrm{mM} \mathrm{NaCl}, 24 \mathrm{mM}$ $\mathrm{NaHCO}_{3}, 2.5 \mathrm{mM} \mathrm{KCl} 1.2 \mathrm{mM} \mathrm{NaH}_{2} \mathrm{PO}_{4}, 2 \mathrm{mM} \mathrm{MgSO}_{4}, 2 \mathrm{mM} \mathrm{CaCl}_{2}, 5 \mathrm{mM}$ HEPES, and $12.5 \mathrm{mM}$ glucose. Patch pipettes, pulled with a tip resistance of 6-8 $\mathrm{M} \Omega$, were filled with internal solution (130 $\mathrm{mM} \mathrm{K}^{+}$ gluconate, $0.3 \mathrm{mM}$ EGTA, $4 \mathrm{mM} \mathrm{KCl}, 10 \mathrm{mM}$ HEPES, $4 \mathrm{mM}$ ATPMg, and $0.3 \mathrm{mM} \mathrm{Na}_{2}-\mathrm{GTP}, \mathrm{pH}$ 7.4). Recording data were filtered at $2 \mathrm{kHz}$, sampled at $10 \mathrm{kHz}$ with a Digidata 1440 and Clampex 10.6 (Molecular Devices, USA) and acquired and analyzed using pClamp10.6 software (Molecular Devices, USA).

\section{Golgi-Cox Staining}

Golgi-Cox staining was used to observe the morphology of neuronal dendrites and synaptic plasticity as previously reported $[32,33]$. The animals were anesthetized and perfused with a $0.9 \%$ sterile saline solution. The brains were removed and stained with Golgi-Cox solution (consisting $5 \%$ potassium chromate, $5 \%$ potassium dichromate, and $5 \%$ mercuric chloride) and stored at RT in the dark for $2-3$ days. After that, the solution was replaced with fresh Golgi-Cox solution for another two weeks. Then the brains were kept in a $25-30 \%$ sucrose solution for two days in order to reduce the tissue fragility during the sectioning process. After collection of 100-200- $\mu \mathrm{m}$-thick coronal slices using a vibratome (Leica, VT1000s, Germany), the brain sections were washed in deionized water for 1 min, placed in $50 \% \mathrm{NH} 4 \mathrm{OH}$ and subsequently in fixing solution (Kodak; Rochester, NY, USA) for $30 \mathrm{~min}$. The sections were subsequently immersed in $5 \%$ sodium thiosulfate for $10 \mathrm{~min}$. After being rinsed with distilled water, the slices were dehydrated using increasing concentrations of ethanol. All sections were photographed under the bright field of a confocal microscope (FV1000, Olympus) with an excitation wavelength of $405 \mathrm{~nm}$. Images were taken by z-stack scanning, and then set the visible light to green.

\section{Immunochemistry}

At the time of sacrifice, animals were perfused intracardially with cold $4 \%$ paraformaldehyde. Frozen brain sections were collected at $30 \mu \mathrm{m}$ intervals from the region between $-2.6 \mathrm{~mm}$ and $-4.1 \mathrm{~mm}$ from bregma and prepared for immunofluorescence. In parallel, 5- $\mu \mathrm{m}$-thick paraffin sections from the same region were prepared for amyloid- $\beta$ ( $A B)$ immunohistochemistry. All the sections were etched with PBS solution containing $20 \%$ methanol and $3 \% \mathrm{H}_{2} \mathrm{O}_{2}$ for 20 min, and blocked with PBS solution containing $0.1 \%$ Triton X-100 and $10 \%$ serum from the same species as the secondary antibody for $30 \mathrm{~min}$. A battery of primary antibodies, including purified anti- $\beta$-Amyloid, 1-16 antibody (1:500) (Biolegend, SIG-39320, San 
Diego, CA, USA), and mouse anti-NeuN (1:100) (Abcam, Ab104224, California, USA) were diluted in blocking solution and incubated with sections overnight at $4{ }^{\circ} \mathrm{C}$. After incubation, the samples were washed in PBS and then incubated with a corresponding secondary antibody solution for $2 \mathrm{~h}$ at RT. The following secondaries were used: Biotinylated anti-mouse [heavy- and light-chains $(\mathrm{H}+\mathrm{L})$; BA-2000; Vector Lab]; A488 anti-mouse IgG (1:500) (Thermo Fisher Scientific, A-32790, New York, USA). DAPI (SigmaAldrich, 32670, California, USA) was used for cell nuclei counterstaining, and the peroxidase reaction was developed using a DAB substrate. All immunostained sections were photographed under a confocal microscope (Olympus, FV10-ASW, Japan) or a light microscope (Leica, DMi8, Germany), and analyzed using Image J Pro Plus V 6.0 (Bethesda, Maryland, USA).

\section{Thioflavin S staining}

Thioflavin $S$ staining was performed in order to detect $A \beta$ plaques in brain tissue as previously described $[34,35]$. Briefly, animals were perfused with a $4 \%$ paraformaldehyde solution and brain tissue was removed, followed by preparation of paraffin-embedded samples. After dewaxing, 5 - $\mu \mathrm{m}$-thick paraffin sections were prepared and then washed three times for 5 min each. DAPI was added and incubated with the tissue sections for $8 \mathrm{~min}$. A $0.3 \%$ thioflavin S solution was conFig.d in $50 \%$ alcohol ( $\mathrm{v} / \mathrm{v})$ and filtered, and then incubated for $8 \mathrm{~min}$ at RT. The sections were washed in $80 \%$ alcohol for $10 \mathrm{sec}$ at RT and stained with $0.3 \%$ thioflavin $\mathrm{S}$ solution twice for 10 sec each. Subsequently, the sections were rinsed with distilled water one last time. Then, the sections were then coverslipped with neutral balsam. Images were captured with a confocal microscope (Olympus, FV10-ASW, Japan), and analyzed using ImageJ Pro Plus V 6.0 (Bethesda, Maryland, USA).

\section{Western blotting}

Cell samples from the APPwt, APPswe+ PBS, APPswe+ EXO groups ( $n=3$ per group) and hippocampal tissues at seven days, four weeks or 12 weeks after MSC-EXO administration from animals belonging to naive, $A D+$ saline and $A D+E X O$ groups ( $n=5$ per group per time point) were dissected for Western blotting. Samples were lysed in Radio-Immunoprecipitation Assay (RIPA) Lysis Buffer (Beyotime, P0013B, Shanghai, China) containing a 1:100 (v/v) ratio of a protease inhibitor cocktail and a phosphatase inhibitor cocktail (Millipore, MA, USA). Total protein samples were quantified using the BCA protein assay (Beyotime, P0012, Shanghai, China), and then normalized protein samples were separated via sodium dodecyl sulfate-polyacrylamide gel, and transferred to polyvinylidene fluoride membranes (Millipore, MA, USA). Membranes were blocked in tris-buffered saline (TBS) containing $0.1 \%(\mathrm{v} / \mathrm{v})$ Tween 20 (TBST) and $5 \%(\mathrm{w} / \mathrm{v})$ nonfat milk at RT for $2 \mathrm{~h}$. Membranes were incubated with primary antibodies including Nrf2 (1:1000) (Abmart, T55136S, Shanghai, China), hemeoxygenase-1 (HO-1, 1:1000) (Proteintech, 10701-1-AP, Illinois, USA), kelch-like ECH-associated protein 1 (Keap1, 1:1000) (Proteintech, 10503-2-AP, Illinois, USA) NQ01 (1:1000) (Abmart, T56710M, Shanghai, China), INOS (1:1000) (Proteintech, 18985-1-AP, Illinois, USA) or $\beta$-actin (1:100000) (Abclonal, AC026, Wuhan, China) overnight at $4{ }^{\circ} \mathrm{C}$, followed by three TBST washes. Membranes were then incubated with HRP-conjugated anti-mouse secondary antibodies (Cat. CW0102S, CWBIO CompanyLimited), or HRP-conjugated anti-rabbit secondary antibodies (Cat. EK020, 
Zhuangzhi Biotech Company Limited) at RT for $2 \mathrm{~h}$. Images were taken using a Bio-Rad imaging system (Bio-Rad, Hercules, California, USA) and analyzed by the Quantity One software package (West Berkeley, California, USA).

\section{Morris Water Maze (MWM)}

The Morris water maze was used to test spatial memory and cognitive function of naive, $A D+$ saline and AD+EXO group ( $n=8 \sim 10$ per group) as previously described [36,37]. The tests were conducted in a water maze drum filled with milky white water dyed by edible pigment at $20^{\circ} \mathrm{C} \pm 1{ }^{\circ} \mathrm{C}$. The testing room was kept quiet during the experiment to minimize the interference of experimenter on mice. Spatial learning sessions were performed over four consecutive days with four trials per day. A small escape platform was submerged $1-2 \mathrm{~cm}$ under water surface. The mice were gently placed into the water, facing the wall, at the corresponding entry point in a quasi-random fashion to prevent strategy learning, free to find the platform. Each trial lasted until the animal found the platform or for a maximum of $60 \mathrm{~s}$. If the mice failed to find the platform within $60 \mathrm{~s}$, they were guided to the platform for another $30 \mathrm{~s}$. After every trial, the mice were placed in a clean, warm environment and given food and water to replenish their energy. A $60 \mathrm{~s}$ probe test was conducted $24 \mathrm{~h}$ from the last learning session, during which, the hidden platform that was present for the first four days was removed. Mice were allowed to swim freely in the water for $60 \mathrm{~s}$. A tracking system (Morris2.8.1) was used that automatically recorded and analyzed the latencies to reach the platform, the activity time, the percentage of the total time (\%, PT) in the target quadrant, and the frequency of mice crossing the platform.

\section{Novel object recognition test (NORT)}

The NORT was conducted to measure recognition memory as previously described [38]. Briefly, mice were individually placed in a square open field apparatus with a side length of $44 \mathrm{~cm}$, free to explore for $5 \mathrm{~min}$ in the habituation session, and then returned to their home cage. After each experiment, the open filed was thoroughly cleaned with $75 \%(\mathrm{v} / \mathrm{v})$ ethanol to minimize olfactory cues before the next mouse entered the open field. In the familiarization phase performed $24 \mathrm{~h}$ after the first session, two identical objects were placed in two opposite areas of the apparatus $10 \mathrm{~cm}$ away from the wall, and the mice were again placed in the field with their head positioned opposite the objects to explore for $10 \mathrm{~min}$. Twenty-four $\mathrm{h}$ later, the test session was conducted. One of the objects was replaced with a novel one and mice were placed in the arena containing one familiar and one new object to explore for another $10 \mathrm{~min}$. Their behavior and exploring time were recorded by a video tracking system for analysis. A discrimination index was calculated as follows: Discrimination index $=$ (time on novel - time on familiar) $/$ (time on novel + time on familiar). All operations were conducted between 8 a.m. and 5 p.m.

\section{Statistical analysis}

All the data are presented as Mean \pm SEM. Multiple comparisons were analyzed using one-way analyses of variance (ANOVA) or unpaired, two-tailed Student's t-test. A repeated measures ANOVA was carried out to analyze the differences in escape latency among groups after Bonferroni posttest using SPSS 24.0.0 
and GraphPad Prism 6 software (GraphPad Prism, USA). P values of less than 0.05 were considered statistically significant.

\section{Results}

\section{MSC-EXO characterization and tracking in mice}

MSCs were obtained from Wharton's jelly as previously described. A flow cytometry analysis showed that the 3rd passage of harvested cells presented strong expression of stromal markers, including CD1105, CD44, CD29 and CD90, and almost negative expression of hematopoietic lineage markers CD 45 and CD34 (Fig. 1A) as described for the specific criteria of MSCs [39]. Characterization of the MSC-EXO was performed by Western blotting, NTA, and TEM. The protein assay results showed that the extracellular vesicles (EVs), isolated from the culture medium of MSCs, expressed exosomal markers such as CD63, CD9, and TSG101 (Fig. 1B), while the size of nanovesicles was distributed from $40 \sim 160 \mathrm{~nm}$ (Fig. 1C) and the morphology presented a typical cup-shape (Fig. 1D). Together, these data suggest that these EVs are consistent with the typical characteristics of exosomes. Although multiple studies have demonstrated the permeability of exosome to the blood-brain barrier and targeting of neural cells in neurological disorders [40, 41], we used CM-A594 labeling to track the MSC-EXO in APP/PS1 mice to ensure the vesicle delivery. Twenty-four $h$ after a tail vein injection of MSC-EXO, fluorescent staining showed that labelled exosomes were observed in the cytoplasm of neuronal cells within the hippocampus.

\section{Tail vein injection of MSC-EXO reduces $A \beta$ aggregation in APP/PS1 mice}

$A \beta$ is believed to be a crucial and primary factor in triggering progressive loss of neuronal function in $A D$ [42]. Therefore, we performed histochemistry, thioflavin staining and Western blotting to examine $A \beta$ aggregation in APP/PS1 mice. The histochemistry results showed that, compared to the naive control, $A \beta$ deposition was increased in APP/PS1 mice as evidenced by the size and IOD value of 6E10 (evidencing deposited amyloid plaques) staining in the hippocampus (Fig. 2B, D, P < 0.01) and cortex (Fig. 2C, E, P< 0.01). After MSC-EXO treatment, a reduction in $6 E 10$ expression was observed in $A D+E X O$ mice that was not observed in the $A D+$ saline mice (Fig. $2 B-D, P<0.05$ ). Thioflavin staining was further used to probe the $A \beta$ protein deposition in the experimental groups, and consistent with above results, the positive area (green) and mean fluorescence intensity in the hippocampus (Fig. 2G, I, P<0.05) and cortex (Fig. 2H, J, P $<0.05$ ) of the $A D+E X O$ group were significantly lower than those observed in the $A D+$ saline group. Additionally, a protein assay (Fig. $2 \mathrm{~K}$ ) showed that compared to naive control, an increase of $A \beta$ expression was observed in APP/PS1 + saline mice (Fig. $2 \mathrm{~L}, \mathrm{P}<0.001$ ), whereas exosome therapy remarkably decreased $(P<0.05)$ expression of $A \beta$ in the AD model ( $A D+E X O$ group). Together, these results imply that MSC-EXO inhibit the aggregation of A observed in APP/PS1 mice

\section{MSC-EXO administration repairs the hippocampal neuronal morphology alterations in mice}

Synaptic morphology and plasticity are critical for neuronal function and are known to be associated with the memory impairment in AD [43]. Herein, we examined the dendritic processes and spines of 
hippocampal pyramidal neurons using Golgi staining at 3 months after exosome treatment. The representative images displayed obvious morphological differences including dendritic complexity (Fig. 3A), three-dimensional (3D) reconstruction of spines, (Fig. 3B) and dendritic phenotype (Fig. 3C) between the naive, $A D+$ saline, and $A D+E X O$ groups. $3 D$ reconstruction of dendrites showed a reduction of total dendritic density (Fig. 3D, $P<0.0001$ ), and filopodia/mushroom/long thin dendrite spine density (Fig. 3E-G, $\mathrm{P}<0.01$ ) in hippocampal pyramidal neurons in APP/PS1 mice compared to naive controls. Additionally, after neuronal phenotype sholl analysis, we found that APP/PS1 mice had lower total spine length (Fig. $3 \mathrm{l}, \mathrm{P}<0.0001)$ and lower dendritic intersections of basal dendrites $(-40-180 \mu \mathrm{m})(\mathrm{Fig} .3 \mathrm{~J})$ than naive mice. Remarkably, these morphological changes in pyramidal neurons were reversed in the AD + EXO group compared to the AD + saline group (Fig. 3D-G and I-J, $P<0.05$ ). Together, these results suggest that the morphological alterations observed in hippocampal neurons of APP/PS1 mice are restored by MSC-EXO therapy.

\section{Exosome therapy restores neuronal excitability and mitochondrial changes in the AD model}

To investigate the restoration of MSC-EXO on neuronal function in the AD model, given that hippocampal CA1 pyramidal neurons are responsible for learning, memory and cognition [44], the excitability of these cells was detected by whole cell patch clamp recording (Fig. 4A). After analyzing the waveform of pyramidal neurons (Fig. 4B), we found a significant increase in half-width (Fig. 4C, $P<0.05$ ) and a significant decrease in membrane potential (Fig. 4D, $P<0.05$ ) and frequency (Fig. $4 E, P<0.01$ ) of action potentials in the $A D+$ saline group compared to the naive group, whereas EXO treatment significantly reversed (Fig. 4C-E, $\mathrm{P}<0.05$ ) these potential changes, suggesting that MSC-EXO treatment restores the excitability of hippocampal CA1 pyramidal neurons in APP/PS1 mice. Importantly, mitochondria are essential for the maintenance of proper function and quality control in hippocampal neurons [45]. We therefore examined the ultrastructure (Fig. 4F) and injury markers (Fig. 4G) of mitochondria in the experimental groups. As shown in TEM images (Fig. 4F), the mitochondria in hippocampal neurons of AD mice exhibited obvious swelling and vacuolation (red arrow) compared with the naive group, while a trend toward normal mitochondrial structure was observed in the AD + EXO group. Moreover, compared with the naive group, the altered mitochondrial fission / fusion was typified by an increase in COX $\otimes$ (Fig. $4 \mathrm{H}, \mathrm{P}<$ 0.001), Tom20 (Fig. 4I, $\mathrm{P}<0.001$ ), and FIS1 (Fig. 4J, $\mathrm{P}<0.001$ ) expression in the hippocampal tissue of AD mice. Notably, after MSC-EXO treatment, these protein markers were found to be reduced in comparison to the $A D+$ saline group (Fig. $4 \mathrm{H}-\mathrm{J}, \mathrm{P}<0.01$ ). Combined, these results suggest that exosometherapy ameliorates the mitochondrial changes observed in APP / PS1 mice.

\section{MSC-EXO treatment ameliorates calcium transients in A -induced primary culture of hippocampal neurons}

It is believed that calcium imbalance induced by amyloid $A \beta$ drives the synaptic plasticity and neuronal loss observed in $A D$ [46]. Therefore, we assessed for calcium signaling alterations using an $A \beta$-induced primary culture of hippocampal neurons. Calcium imaging revealed differences in the fluorescence properties of hippocampal neurons among the experimental groups (Fig. 5A). Following ATP stimulation, 
the first phase calcium response consisted of a sharp peak in calcium signaling, followed by a second phase response of a slowly declining intracellular calcium concentration in each group (Fig. 5B). The statistical analysis revealed that, compared to the control group, $A \beta$ induction resulted in a reduced amplitude of intracellular calcium transients (Fig. $5 \mathrm{C}, \mathrm{P}<0.0001$ ) in the primary culture of hippocampal neurons after adding ATP, whereas MSC-EXO treatment significantly increased the calcium influx compared to the $A \beta+P B S$ group (Fig. $5 C, P<0.05$ ). We also found a slower change in the response rise time (Fig. 5D, $P<0.0001$ ) as well as the decay time (Fig. 5E, $P<0.0001$ ) in the $A \beta+P B S$ group in compared to the control group. Remarkably, exosome-therapy $(A \beta+E X O)$ reversed the rate of calcium transients observed in the A $\beta+P B S$ group (Fig. $D, E, P<0.0001$ ). Together, these data indicate that MSCEXO treatment ameliorates the alterations in calcium transients in A $\beta$-induced primary hippocampal neurons, further supporting the therapeutic potential of MSC-EXO on neuronal dysfunction.

\section{Exosomes Treatment Improves Cognitive Deficits In The App/ps1 Mice}

After MSC-EXO administration for three months, the MWM and NORT were performed to assess for cognitive deficits. Analysis of the MWM (Fig. 6A) showed that there was no significant difference in the swim speed (Fig. 6B, $P>0.05$ ) among the experimental groups, which excluded the influence of dyskinesia on the results. Compared to the naive group, the learning and memory impairments in the AD + saline group were characterized by a longer time of escape latency (Fig. 6C, P < 0.0001) and a decrease of platform crossings (Fig. 6D, P<0.01) and percent time (PT) in the target quadrant (Fig. 6E, P< 0.05). Whereas an indication of behavioral improvements (including escape latency, platform crossings and PT in the target quadrant) were observed in the $A D+E X O$ group (Fig. $6 C-E, P<0.05$ ) compared to the $A D+$ saline group. Additionally, recognition memory function was examined using the NORT. We found that compared to the naive group, the $A D+$ saline group displayed an inability for novel object discrimination (N, Fig. 6F) as they spent a similar percentage of time exploring the familiar and novel objects. Notably, animals in $A D+E X O$ group showed an increased discrimination index compared to the $A D+$ saline group (Fig. 6G, P < 0.05), suggesting that the recognition memory function in APP / PS1 mice was ameliorated

by EXO treatment. Together, these results indicate that MSC-EXO treatment improves the cognitive deficits observed in APP/PS1 mice.

\section{Oxidative defense system is associated with the exosome therapy in APP/PS1 mice}

Oxidative stress is believed to be a cause of neuronal degeneration in Alzheimer's disease, while Nrf2 play a pivotal role in the mediation of oxidative stress $[47,48]$. We first investigated whether Nrf2 signaling participates in the action of MSC-EXO in APP/PS1 mice. As shown in Fig. 7A, compared with agematched control mice, the AD mice (AD + saline) displayed a remarkably higher level of Nrf2, HO-1, NQO1, iNOS (Fig. 7B, D-F, $P<0.001$ ) and a lower level of Keap1 protein expression (Fig. 7C, $P<0.0001$ ), while exosomes treatment reversed the expression of these proteins (Fig. 7B-F, $\mathrm{P}<0.05$ ), suggesting the involvement of the Nrf2 signaling pathway in the treatment of AD model. To further confirm the participation of this signaling in the action of MSC-EXO on the hippocampal neurons, AD cell models including the SHSY5Y (APPwt) and SHSY5Y (APPswe) cell lines were employed in an in vitro study [27]. 
As can be seen in Fig. 7G, Nrf2 signaling (Nrf2, Keap1, HO-1, NQ01) and INOS exhibited variations similar to those observed in the in vivo studies between the APPwt and APPswe + PBS group (Fig. 7H-L, P<0.01). Additionally, MSC-EXO administration significantly reduced the expression of Nrf2, HO-1, NQO1, iNOS and increased the expression of Keap1 in the APPswe + EXO group (Fig. 7H-L, P<0.05) compared to the APPswe + PBS group, indicating that Nrf2 signaling participates in the action of MSC-EXO on hippocampal neurons in AD. Notably, Nrf2 expression was significantly increased after exosomes treatment compared to the naive or APPwt group, which may suggest that MSC-EXO activate the Nrf2 pathway in the AD model.

\section{Discussion}

Our results show that MSC-EXO treatment inhibits the deposition of A $\beta$ protein observed in the brain of $A D$ mice as well as ameliorates the deficits in neuronal structure and function typified by the morphology alterations, mitochondrial changes, excitability restoration, calcium transients, and associated cognitive repairments observed in the AD cell and mouse models. Additionally, the present data demonstrated that the Nrf2 signaling pathway participates in the actions of MSC-EXO on neuronal dysfunction in AD using cell and mouse models. Together, these results suggest that MSC-EXO can represent a promising nanotherapeutic agent for the treatment of $A D$.

It is well known that $A \beta$ protein deposition is the most typical pathological feature in $A D$ patients and animal models. To ensure the administered MSC-EXO produced therapeutic effects on APP/PS1 mice, we performed a systematic investigation of $A \beta$ aggregation using an $A \beta$ indicator (6E10), Thioflavin staining, and western blotting. Consistent with a previous study [17], exosome-therapy reduced the expression of $A \beta$ related indications, implying the positive effects of MSC-EXO on A $\beta$ aggregation in APP/PS1 mice brain. Importantly, cognitive and memory impairments are one of the main clinical manifestations observed in AD patients [4]. The MWM and NORT tests were used to detect the cognitive decline in APP/PS1 mice, and our results showed that EXO treatment improved their behavioral performance compared to the saline group, suggesting that the cognitive deficits can be improved by MSC-EXO. Together, the above results indicate that MSC-EXO show markable therapeutic effects on behavior in APP/PS1 mice.

Among the multiple features of $A D$ initiation and progression, including $A \beta$ deposition, neuroinflammation, oxidative stress, and abnormal neurogenesis, neuronal dysfunction is believed to be the ultimate cause of cognitive decline, and the hippocampus is one of the most affected areas in $A D$ patients [49]. Therefore, the present study focused on the reconstruction of hippocampal neurons by MSC-EXO treatment. Synaptic transmission provides the physiological, cellular, and molecular mechanisms for cognitive function, while synaptic plasticity is reflected by dendritic density and complexity in neuronal cells. Herein, extensive loss of synapses and dendritic spines is one of the important pathological features of AD [50]. In the present study, impairments of synaptic plasticity within hippocampal neurons were observed in AD mice. After exosome-therapy, the morphological changes in spine density and dendritic intersections implied that the structural impairments in hippocampal 
pyramidal neurons can be restored by MSC-EXO in APP/PS1 mice. Accordingly, compared with the AD + saline group, the increased performance of the AD + EXO group suggests that EXO treatment facilitates the recovery of learning and memory in AD mice. Collectively, these data suggest that MSC-EXO facilitate the reconstruction of neuronal structure thus ameliorating the associated cognitive decline observed in APP/PS1 mice.

Moreover, abnormal excitability is a well-known alteration that occurs in neurodegenerative diseases such as AD [51]. Our whole-cell patch-clamp experiment is consistent with this as the electrophysiological activity of hippocampal neurons was altered in APP / PS1 mice, evidenced by a decreased frequency of Aps and membrane potentials. Whereas MSC-EXO administration reversed this alteration in neuronal excitability suggesting that EXO treatment restores the electrophysiological function of hippocampal neurons compared to the AD + saline group. Importantly, mitochondria generated ATP is essential for the excitability and survival of neurons, which functions to regulate calcium influx, synaptic plasticity, and redox signaling [52-54]. Thus, calcium imaging performed to detect the calcium transients in cultured primary hippocampal neurons in response to $A \beta$ stimulation. MSC-EXO treatment ameliorated the alterations in calcium transients observed in the A $\beta+P B S$ group as evidenced by the amplitude of signaling, rise time and decay time. Previous studies have reported that mitochondrial dysfunction is involved in the pathogenesis of most nervous system diseases including AD [55]. Here, we used TEM to detect the mitochondrial ultrastructure and protein assay to examine mitochondrial fission / fusion in the experimental groups. Compared to the saline group, the ameliorated mitochondrial ultrastructure and decreased $\mathrm{COX} \otimes$, Tom 20 and FIS1 expression observed in AD + EXO group, indicated that exosomes administration improves the mitochondrial dysfunction in APP / PS1 mice. Together, these results suggest that the abnormalities in neuronal structure and dysfunction observed in $A D$ can be restored by MSC-EXO, which provides new evidence for the nanotherapeutic action of EXO in AD mice.

Furthermore, hippocampal neurons are highly susceptible to oxidative stress in AD patients and animal models, and the redox imbalance is considered to be a prominent factor of neuronal damage in the brain [56]. Taking this into consideration, examination of the oxidative defense system may reveal the mechanism(s) underlying MSC-EXO therapeutic action on hippocampal neurons in AD. Furthermore, our previous study indicated that MSC-EXO show antioxidant activity as they contained enriched functional agents such as nucleic acids, proteins and enzymes that regulate redox reactions [23]. Antioxidants also have great potential in the treatment of neurodegenerative diseases and have been shown to improve learning and memory deficits in $A D$ mice $[57,58]$. Among the multiple molecular pathways implicated in the oxidative defense system, $\mathrm{Nrf} 2$ is an essential element for the regulation of oxidative responses in neuronal degeneration [59]. Under physiological conditions, Nrf2 interacts with the cytoplasmic protein Keap1, whereas it is isolated from Keap1 and translocated into the nucleus under stress conditions [60, 61]. Numerous studies have indicated that Nrf2 can activate transcription of its target genes $\mathrm{HO}-1$ and NQ01, which then exerts a cytoprotective effect against neuronal damage [62]. In the present study, compared with AD + saline group, MSC-EXO treatment resulted in a decrease of Nrf2, HO-1, NQ01, iNOS (indicative of oxidative damage), and an increase of Keap1 expression in hippocampal tissues, suggesting that the Nrf2 signaling pathway participates in the therapeutic action of exosomes in the APP 
/ PS1 mouse brain. Moreover, these changes were also observed in the AD cell models (SHSY5Y, APPswe) after EXO administration, which further supports the involvement of the Nrf2 defense system in the therapeutic effects of MSC-EXO in AD mice. Combined, these results suggest that the therapeutic effects of MSC-EXO on hippocampal neuronal dysfunction might be regulated by Nrf2 signaling pathway.

In conclusion, the present study shows that MSC-EXO treatment ameliorates the hippocampal neuronal structural and functional deficits observed in APP/PS1 transgenic mice, and that the therapeutic mechanism is associated with the Nrf2 defense system in vitro and in vivo, suggesting that MSC-EXO can serve as functional nanotherapeutic agents in the treatment of AD.

\section{Declarations}

\section{Ethics Approval}

This experiment was approved by the Guideline of the Ethical Committee of the Xi'an Central Hospital, Xi'an Jiaotong University (No. 20180715-1), all animal procedures were performed in accordance with the guidelines for the Care and Use of Laboratory Animals of the National Institutes of Health.

\section{Conflict of Interest}

The authors declare that they have no conflict of interests.

\section{Funding}

This work was supported by grants from the National Natural Science Foundation of China (Nos. 81871084 and 81627806), Military Upgrade Plan of Xijing Hospital, Fourth Military Medical University (2016TSB021), Discipline Promotion Program of Xijing Hospital, Fourth Military Medical University (XJZT19MJ22), Novel clinical medical technology and business Projects of Xijing Hospital, Fourth Military Medical University (XJGX15Z08), key R\&D Projects of Shaanxi Province (2018ZDXM-SF-046), and Major Projects of Xi'an Medical Research (201805104YX12SF38(1).

\section{Authors' contributions}

All authors contributed to the study conception and design. QL, WL, and AB designed the research. Material preparation, data collection and analysis were performed by $H W, Y L, J L, T W, Y H$, and $H L$. The first draft of the manuscript was written by HW and QL, and all authors commented on previous versions of the manuscript. All authors read and approved the final manuscript.

\section{References}

1 Lane, C.A., Hardy, J., and Schott, J.M. (2018). Alzheimer's disease. European Journal of Neurology, 25(1), 59-70. https://doi.org/10.1111/ene.13439. 
2 (2020). 2020 Alzheimer's disease facts and figures. Alzheimers Dement, 16, 391-460. https://doi.org/10.1002/alz.12068.

3 Galvin, J.E., Howard, D.H., Denny, S.S., Dickinson, S., and Tatton, N. (2017). The social and economic burden of frontotemporal degeneration. Neurology, 89(20), 2049-2056. https://doi.org/10.1212/wnl.0000000000004614.

4 Scheltens, P., Blennow, K., Breteler, M.M., de Strooper, B., Frisoni, G.B., Salloway, S., et al. (2016). Alzheimer's disease. Lancet, 388(10043), 505-517. https://doi.org/10.1016/s0140-6736(15)01124-1.

5 Serrano-Pozo, A., Frosch, M.P., Masliah, E., and Hyman, B.T. (2011). Neuropathological alterations in Alzheimer disease. Cold Spring Harbor Perspectives in Medicine, 1(1) a006189. https://doi.org/10.1101/cshperspect.a006189.

6 Benilova, I., Karran, E., and De Strooper, B. (2012). The toxic A $\beta$ oligomer and Alzheimer's disease: an emperor in need of clothes. Nature Neuroscience, 15(3), 349-357. https://doi.org/10.1038/nn.3028.

7 Shin, J.Y., Park, H.J., Kim, H.N., Oh, S.H., Bae, J.S., Ha, H.J., et al. (2014). Mesenchymal stem cells enhance autophagy and increase $\beta$-amyloid clearance in Alzheimer disease models. Autophagy, 10(1), 32-44. https://doi.org/10.4161/auto.26508.

8 Elia, C.A., Tamborini, M., Rasile, M., Desiato, G., Marchetti, S., Swuec, P., et al. (2019). Intracerebral Injection of Extracellular Vesicles from Mesenchymal Stem Cells Exerts Reduced $A \beta$ Plaque Burden in Early Stages of a Preclinical Model of Alzheimer's Disease. Cells, 8(9), 1059. https://doi.org/10.3390/cells8091059.

9 Park, H.J., Oh, S.H., Kim, H.N., Jung, Y.J., and Lee, P.H. (2016). Mesenchymal stem cells enhance asynuclein clearance via M2 microglia polarization in experimental and human parkinsonian disorder. Acta Neuropathologica, 132(5), 685-701. https://doi.org/10.1007/s00401-016-1605-6.

10 Lee, H.J., Lee, J.K., Lee, H., Carter, J.E., Chang, J.W., Oh, W., et al. (2012). Human umbilical cord blood-derived mesenchymal stem cells improve neuropathology and cognitive impairment in an Alzheimer's disease mouse model through modulation of neuroinflammation. Neurobiology of Aging, 33(3), 588-602. https://doi.org/10.1016/j.neurobiolaging.2010.03.024.

11 Gao, L.B., Yu, X.F., Chen, Q., and Zhou, D. (2016). Alzheimer's Disease therapeutics: current and future therapies. Minerva Medica, 107(2), 108-113.

12 Labrador-Velandia, S., Alonso-Alonso, M.L., Di Lauro, S., García-Gutierrez, M.T., Srivastava, G.K., Pastor, J.C., et al. (2019). Mesenchymal stem cells provide paracrine neuroprotective resources that delay degeneration of co-cultured organotypic neuroretinal cultures. Experimental Eye Research, 185, 107671. https://doi.org/10.1016/j.exer.2019.05.011. 
13 Burdon, T.J., Paul, A., Noiseux, N., Prakash, S., and Shum-Tim, D. (2011). Bone marrow stem cell derived paracrine factors for regenerative medicine: current perspectives and therapeutic potential. Bone Marrow Res, 2011, 207326. https://doi.org/10.1155/2011/207326.

14 Zhang, Z.G., Buller, B., and Chopp, M. (2019). Exosomes - beyond stem cells for restorative therapy in stroke and neurological injury. Nature Reviews: Neurology, 15(4), 193-203.

https://doi.org/10.1038/s41582-018-0126-4.

15 Chen, C.C., Liu, L., Ma, F., Wong, C.W., Guo, X.E., Chacko, J.V., et al. (2016). Elucidation of Exosome Migration across the Blood-Brain Barrier Model In Vitro. Cellular and Molecular Bioengineering, 9(4), 509529. https://doi.org/10.1007/s12195-016-0458-3.

16 Wang, S.S., Jia, J., and Wang, Z. (2018). Mesenchymal Stem Cell-Derived Extracellular Vesicles Suppresses iNOS Expression and Ameliorates Neural Impairment in Alzheimer's Disease Mice. Journal of Alzheimer's Disease, 61(3), 1005-1013. https://doi.org/10.3233/jad-170848.

17 Santamaria, G., Brandi, E., Vitola, P., Grandi, F., Ferrara, G., Pischiutta, F., et al. (2021). Intranasal delivery of mesenchymal stem cell secretome repairs the brain of Alzheimer's mice. Cell Death and Differentiation, 28(1), 203-218. https://doi.org/10.1038/s41418-020-0592-2.

18 Long, Q., Upadhya, D., Hattiangady, B., Kim, D.K., An, S.Y., Shuai, B., et al. (2017). Intranasal MSCderived $\mathrm{A} 1$-exosomes ease inflammation, and prevent abnormal neurogenesis and memory dysfunction after status epilepticus. Proceedings of the National Academy of Sciences of the United States of America, 114(17), E3536-E3545. https://doi.org/10.1073/pnas.1703920114.

19 Palop, J.J., and Mucke, L. (2016). Network abnormalities and interneuron dysfunction in Alzheimer disease. Nature Reviews: Neuroscience, 17(12), 777-792. https://doi.org/10.1038/nrn.2016.141.

20 Calvo-Rodriguez, M., Kharitonova, E.K., and Bacskai, B.J. (2020). Therapeutic Strategies to Target Calcium Dysregulation in Alzheimer's Disease. Cells, 9(11), 2513. https://doi.org/10.3390/cells9112513.

21 Kamat, P.K., Kalani, A., Rai, S., Swarnkar, S., Tota, S., Nath, C., et al. (2016). Mechanism of Oxidative Stress and Synapse Dysfunction in the Pathogenesis of Alzheimer's Disease: Understanding the Therapeutics Strategies. Molecular Neurobiology, 53(1), 648-661. https://doi.org/10.1007/s12035-0149053-6.

22 Butterfield, D.A., and Halliwell, B. (2019). Oxidative stress, dysfunctional glucose metabolism and Alzheimer disease. Nature Reviews: Neuroscience, 20(3), 148-160. https://doi.org/10.1038/s41583-0190132-6.

23 Wang, T., Jian, Z., Baskys, A., Yang, J., Li, J., Guo, H., et al. (2020). MSC-derived exosomes protect against oxidative stress-induced skin injury via adaptive regulation of the NRF2 defense system.

Biomaterials, 257, 120264. https://doi.org/10.1016/j.biomaterials.2020.120264. 
24 Théry, C., Amigorena, S., Raposo, G., and Clayton, A. (2006). Isolation and characterization of exosomes from cell culture supernatants and biological fluids. Current Protocols in Cell Biology, Chapter 3, Unit 3.22. https://doi.org/10.1002/0471143030.cb0322s30.

25 Roberts-Dalton, H.D., Cocks, A., Falcon-Perez, J.M., Sayers, E.J., Webber, J.P., Watson, P., et al. (2017). Fluorescence labelling of extracellular vesicles using a novel thiol-based strategy for quantitative analysis of cellular delivery and intracellular traffic. Nanoscale, 9(36), 13693-13706. https://doi.org/10.1039/c7nr04128d.

26 Zhang, J., Liu, Q., Chen, Q., Liu, N.Q., Li, F.L., Lu, Z.B., et al. (2006). Nicotine attenuates betaamyloid-induced neurotoxicity by regulating metal homeostasis. FASEB Journal, 20(8), 1212-1214. https://doi.org/10.1096/fj.05-5214fje.

27 Dong, Y., Stewart, T., Bai, L., Li, X., Xu, T., lliff, J., et al. (2020). Coniferaldehyde attenuates Alzheimer's pathology via activation of Nrf2 and its targets. Theranostics, 10(1), 179-200. https://doi.org/10.7150/thno.36722.

28 Stine, W.B., Jr., Dahlgren, K.N., Krafft, G.A., and LaDu, M.J. (2003). In vitro characterization of conditions for amyloid-beta peptide oligomerization and fibrillogenesis. Journal of Biological Chemistry, 278(13), 11612-11622. https://doi.org/10.1074/jbc.M210207200.

29 Fodero, L.R., Mok, S.S., Losic, D., Martin, L.L., Aguilar, M.I., Barrow, C.J., et al. (2004). Alpha7nicotinic acetylcholine receptors mediate an Abeta(1-42)-induced increase in the level of acetylcholinesterase in primary cortical neurones. Journal of Neurochemistry, 88(5), 1186-1193. https://doi.org/10.1046/j.1471-4159.2003.02296.x.

30 Cheli, V.T., Santiago González, D.A., Smith, J., Spreuer, V., Murphy, G.G., and Paez, P.M. (2016). Ltype voltage-operated calcium channels contribute to astrocyte activation In vitro. Glia, 64(8), 1396-1415. https://doi.org/10.1002/glia.23013.

31 Paez, P.M., Spreuer, V., Handley, V., Feng, J.M., Campagnoni, C., and Campagnoni, A.T. (2007). Increased expression of golli myelin basic proteins enhances calcium influx into oligodendroglial cells. Journal of Neuroscience, 27(46), 12690-12699. https://doi.org/10.1523/jneurosci.2381-07.2007.

32 Wang, M., Liu, X., Hou, Y., Zhang, H., Kang, J., Wang, F., et al. (2019). Decrease of GSK-3 $\beta$ Activity in the Anterior Cingulate Cortex of Shank3b (-/-) Mice Contributes to Synaptic and Social Deficiency. Frontiers in Cellular Neuroscience, 13, 447. https://doi.org/10.3389/fncel.2019.00447.

33 Zhang, Y., Xiao, Z., He, Z., Chen, J., Wang, X., and Jiang, L. (2020). Dendritic complexity change in the triple transgenic mouse model of Alzheimer's disease. PeerJ, 8, e8178. https://doi.org/10.7717/peerj.8178. 
34 Rajamohamedsait, H.B., and Sigurdsson, E.M. (2012). Histological staining of amyloid and preamyloid peptides and proteins in mouse tissue. Methods in Molecular Biology, 849, 411-424. https://doi.org/10.1007/978-1-61779-551-0_28.

35 Zhang, M., Qian, C., Zheng, Z.G., Qian, F., Wang, Y., Thu, P.M., et al. (2018). Jujuboside A promotes $A \beta$ clearance and ameliorates cognitive deficiency in Alzheimer's disease through activating Axl/HSP90/PPARy pathway. Theranostics, 8(15), 4262-4278. https://doi.org/10.7150/thno.26164.

36 Davis, K.E., Burnett, K., and Gigg, J. (2017). Water and T-maze protocols are equally efficient methods to assess spatial memory in 3xTg Alzheimer's disease mice. Behavioural Brain Research, 331, 54-66. https://doi.org/10.1016/j.bbr.2017.05.005.

37 Boza-Serrano, A., Ruiz, R., Sanchez-Varo, R., García-Revilla, J., Yang, Y., Jimenez-Ferrer, I., et al. (2019). Galectin-3, a novel endogenous TREM2 ligand, detrimentally regulates inflammatory response in Alzheimer's disease. Acta Neuropathologica, 138(2), 251-273. https://doi.org/10.1007/s00401-01902013-z.

38 Leger, M., Quiedeville, A., Bouet, V., Haelewyn, B., Boulouard, M., Schumann-Bard, P., et al. (2013). Object recognition test in mice. Nature Protocols, 8(12), 2531-2537. https://doi.org/10.1038/nprot.2013.155.

39 Robb, K.P., Fitzgerald, J.C., Barry, F., and Viswanathan, S. (2019). Mesenchymal stromal cell therapy: progress in manufacturing and assessments of potency. Cytotherapy, 21(3), 289-306. https://doi.org/10.1016/j.jcyt.2018.10.014.

40 Wood, M.J., O'Loughlin, A.J., and Samira, L. (2011). Exosomes and the blood-brain barrier: implications for neurological diseases. Therapeutic Delivery, 2(9), 1095-1099. https://doi.org/10.4155/tde.11.83.

41 Yang, T., Martin, P., Fogarty, B., Brown, A., Schurman, K., Phipps, R., et al. (2015). Exosome delivered anticancer drugs across the blood-brain barrier for brain cancer therapy in Danio rerio. Pharmaceutical Research, 32(6), 2003-2014. https://doi.org/10.1007/s11095-014-1593-y.

42 Yang, H., Li, X., Zhu, L., Wu, X., Zhang, S., Huang, F., et al. (2019). Heat Shock Protein Inspired Nanochaperones Restore Amyloid- $\beta$ Homeostasis for Preventative Therapy of Alzheimer's Disease. Adv Sci (Weinh), 6(22), 1901844. https://doi.org/10.1002/advs.201901844.

43 Liu, Y., Bian, H., Xu, S., Shu, S., Jia, J., Chen, J., et al. (2020). Muscone Ameliorates Synaptic Dysfunction and Cognitive Deficits in APP/PS1 Mice. Journal of Alzheimer's Disease, 76(2), 491-504. https://doi.org/10.3233/jad-200188.

44 Naghdi, N., Majlessi, N., and Bozorgmehr, T. (2003). The effects of anisomycin (a protein synthesis inhibitor) on spatial learning and memory in CA1 region of rats hippocampus. Behavioural Brain 
Research, 139(1-2), 69-73. https://doi.org/10.1016/s0166-4328(02)00060-8.

45 Flannery, P.J., and Trushina, E. (2019). Mitochondrial dynamics and transport in Alzheimer's disease. Molecular and Cellular Neurosciences, 98, 109-120. https://doi.org/10.1016/j.mcn.2019.06.009.

46 Pannaccione, A., Piccialli, I., Secondo, A., Ciccone, R., Molinaro, P., Boscia, F., et al. (2020). The $\mathrm{Na}(+) / \mathrm{Ca}(2+)$ exchanger in Alzheimer's disease. Cell Calcium, 87, 102190.

https://doi.org/10.1016/j.ceca.2020.102190.

47 Sims, N.R. (1996). Energy metabolism, oxidative stress and neuronal degeneration in Alzheimer's disease. Neurodegeneration, 5(4), 435-440. https://doi.org/10.1006/neur.1996.0059.

48 Kaspar, J.W., Niture, S.K., and Jaiswal, A.K. (2009). Nrf2:INrf2 (Keap1) signaling in oxidative stress. Free Radical Biology and Medicine, 47(9), 1304-1309. https://doi.org/10.1016/j.freeradbiomed.2009.07.035.

49 Padurariu, M., Ciobica, A., Mavroudis, I., Fotiou, D., and Baloyannis, S. (2012). Hippocampal neuronal loss in the CA1 and CA3 areas of Alzheimer's disease patients. Psychiatr Danub, 24(2), 152-158.

50 Ingelsson, M., Fukumoto, H., Newell, K.L., Growdon, J.H., Hedley-Whyte, E.T., Frosch, M.P., et al. (2004). Early Abeta accumulation and progressive synaptic loss, gliosis, and tangle formation in AD brain. Neurology, 62(6), 925-931. https://doi.org/10.1212/01.wnl.0000115115.98960.37.

51 Kaczorowski, C.C., Sametsky, E., Shah, S., Vassar, R., and Disterhoft, J.F. (2011). Mechanisms underlying basal and learning-related intrinsic excitability in a mouse model of Alzheimer's disease. Neurobiology of Aging, 32(8), 1452-1465. https://doi.org/10.1016/j.neurobiolaging.2009.09.003.

52 Lee, H.U., Yamazaki, Y., Tanaka, K.F., Furuya, K., Sokabe, M., Hida, H., et al. (2013). Increased astrocytic ATP release results in enhanced excitability of the hippocampus. Glia, 61(2), 210-224. https://doi.org/10.1002/glia.22427.

53 Yamamoto, K., Korenaga, R., Kamiya, A., Qi, Z., Sokabe, M., and Ando, J. (2000). P2X(4) receptors mediate ATP-induced calcium influx in human vascular endothelial cells. American Journal of Physiology: Heart and Circulatory Physiology, 279(1), H285-292. https://doi.org/10.1152/ajpheart.2000.279.1.H285.

54 zur Nedden, S., Hawley, S., Pentland, N., Hardie, D.G., Doney, A.S., and Frenguelli, B.G. (2011). Intracellular ATP influences synaptic plasticity in area CA1 of rat hippocampus via metabolism to adenosine and activity-dependent activation of adenosine A1 receptors. Journal of Neuroscience, 31(16), 6221-6234. https://doi.org/10.1523/jneurosci.4039-10.2011.

55 Castellani, R., Hirai, K., Aliev, G., Drew, K.L., Nunomura, A., Takeda, A., et al. (2002). Role of mitochondrial dysfunction in Alzheimer's disease. Journal of Neuroscience Research, 70(3), 357-360. https://doi.org/10.1002/jnr.10389. 
56 Perry, G., Cash, A.D., and Smith, M.A. (2002). Alzheimer Disease and Oxidative Stress. Journal of Biomedicine \& Biotechnology, 2(3), 120-123. https://doi.org/10.1155/s1110724302203010.

57 Rahman, M.H., Akter, R., and Chowdhury, M.A.R. (2020). Antioxidant as Talented Drug Applicants for the Treatment of Alzheimer's Disease and their Molecular Mechanism Insights. Current Pharmaceutical Design, 26, 1-14. https://doi.org/10.2174/1381612826666201102102810.

58 Wang, C.Y., Wang, Z.Y., Xie, J.W., Wang, T., Wang, X., Xu, Y., et al. (2016). DI-3-n-butylphthalideinduced upregulation of antioxidant defense is involved in the enhancement of cross talk between CREB and Nrf2 in an Alzheimer's disease mouse model. Neurobiology of Aging, 38, 32-46. https://doi.org/10.1016/j.neurobiolaging.2015.10.024.

59 Calkins, M.J., Johnson, D.A., Townsend, J.A., Vargas, M.R., Dowell, J.A., Williamson, T.P., et al. (2009). The Nrf2/ARE pathway as a potential therapeutic target in neurodegenerative disease. Antioxid Redox Signal, 11(3), 497-508. https://doi.org/10.1089/ars.2008.2242.

60 Nguyen, T., Nioi, P., and Pickett, C.B. (2009). The Nrf2-antioxidant response element signaling pathway and its activation by oxidative stress. Journal of Biological Chemistry, 284(20), 13291-13295. https://doi.org/10.1074/jbc.R900010200.

61 Sun, Z., Zhang, S., Chan, J.Y., and Zhang, D.D. (2007). Keap1 controls postinduction repression of the Nrf2-mediated antioxidant response by escorting nuclear export of Nrf2. Molecular and Cellular Biology, 27(18), 6334-6349. https://doi.org/10.1128/mcb.00630-07.

62 Loboda, A., Damulewicz, M., Pyza, E., Jozkowicz, A., and Dulak, J. (2016). Role of Nrf2/HO-1 system in development, oxidative stress response and diseases: an evolutionarily conserved mechanism. Cellular and Molecular Life Sciences, 73(17), 3221-3247. https://doi.org/10.1007/s00018-016-2223-0.

\section{Figures}


A
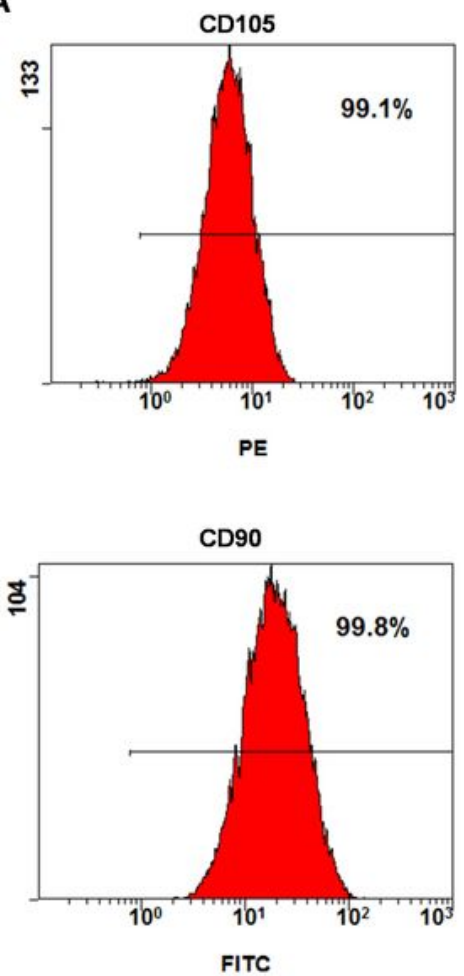

B

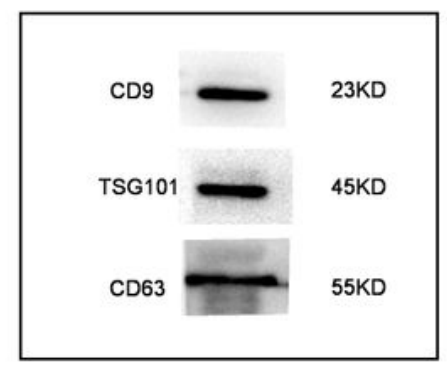

E
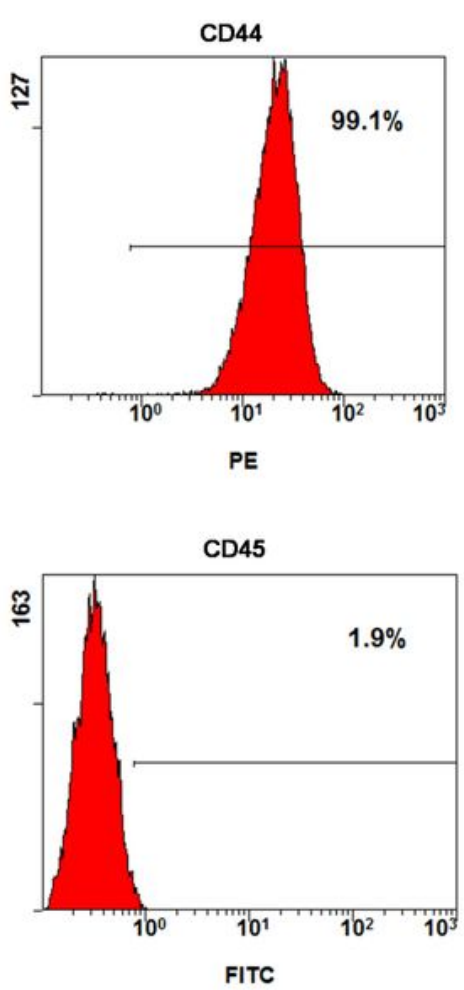

C

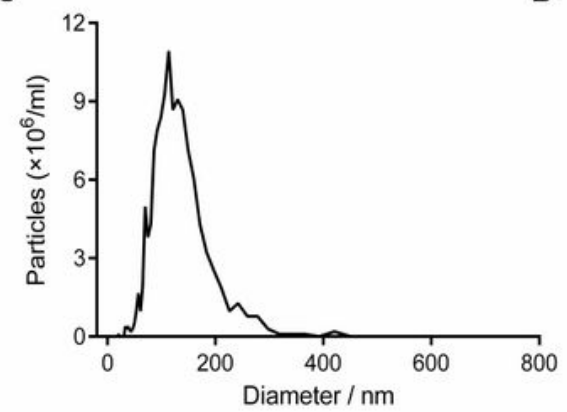

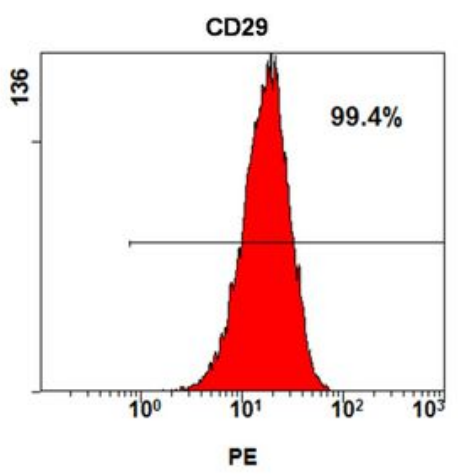

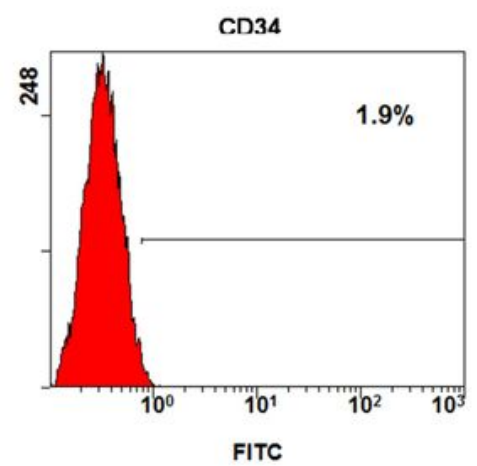

D

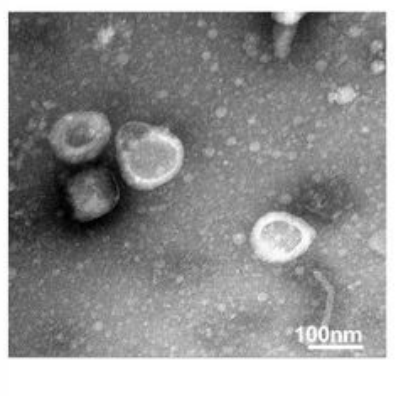

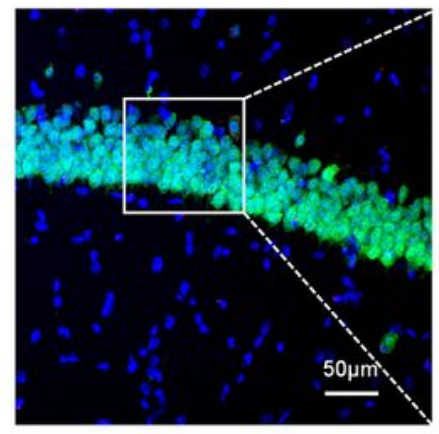

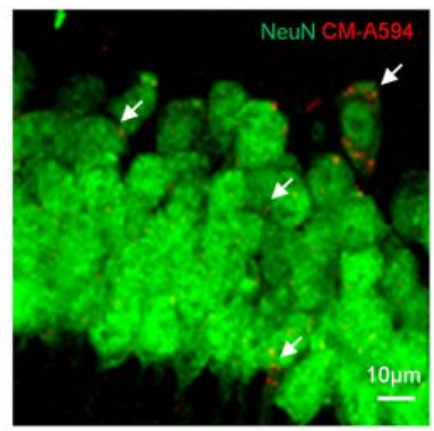

\section{Figure 1}

MSC-EXO characterization and tracking in mice. (A) A flow cytometry analysis of surface biomarkers of MSC. (B) Representative picture of Western blotting for exosomes biomarker protein expression. (C) Size distribution of MSC-EXO. (D) The morphology of MSC-EXO is shown in the TEM image. (E) Representative images show that MSC-EXO were incorporated into hippocampal neurons in vivo. The square area 
appears with higher magnification in the image on the right. The presence of MSC-EXO nanoparticles (red) within $\mathrm{NeuN}+$ neurons (arrowheads).

A
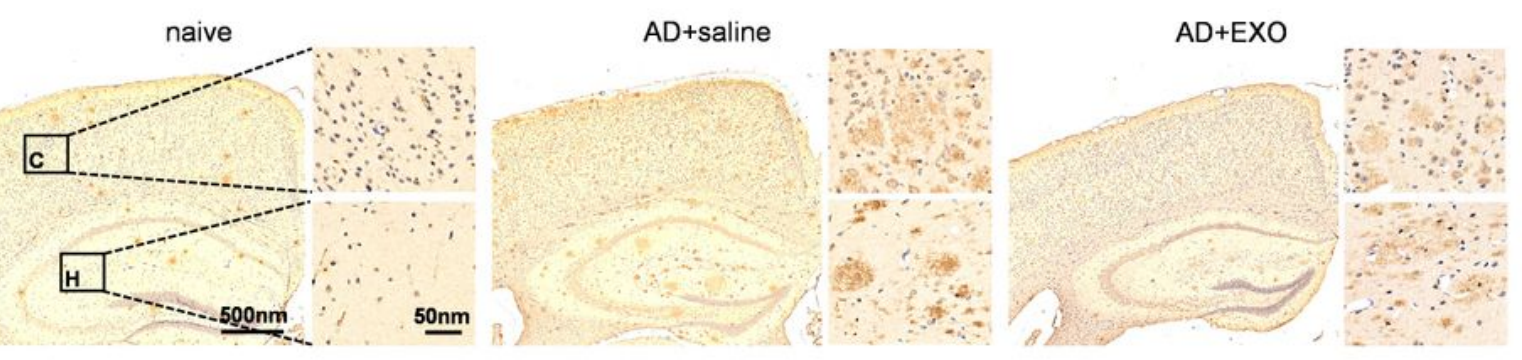

B

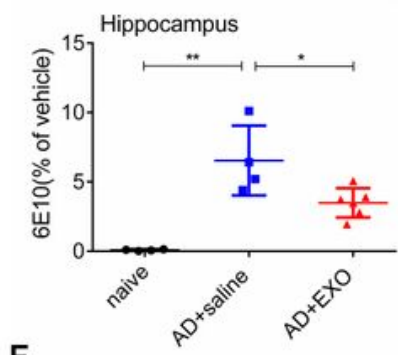

F

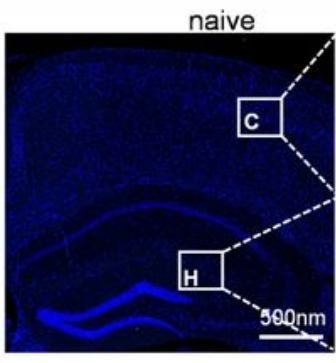

G

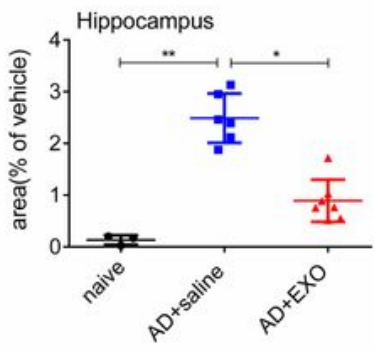

K

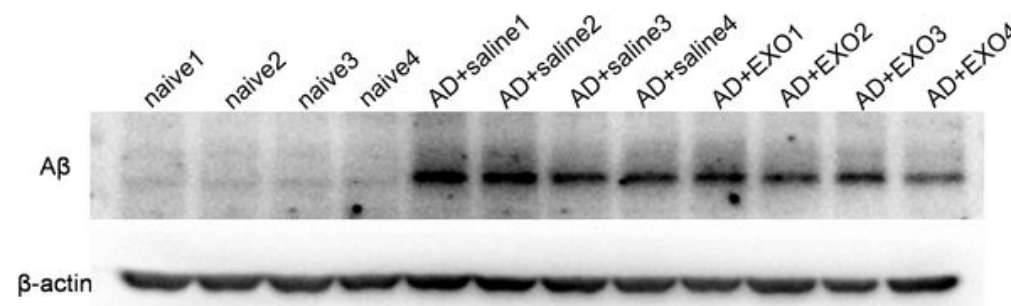

D

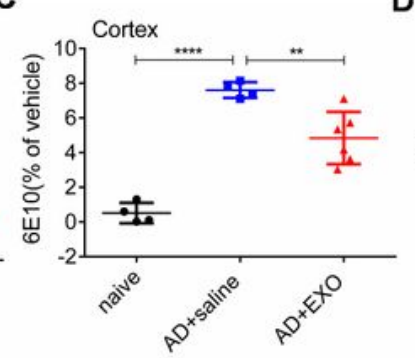

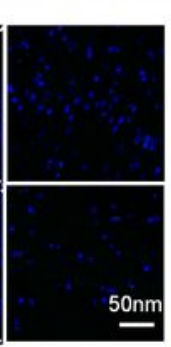

H
AD+saline

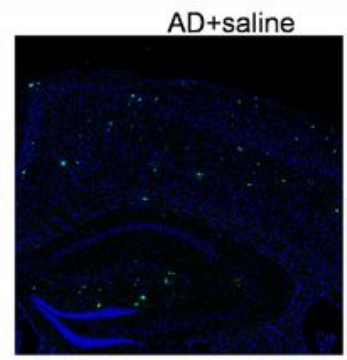

I

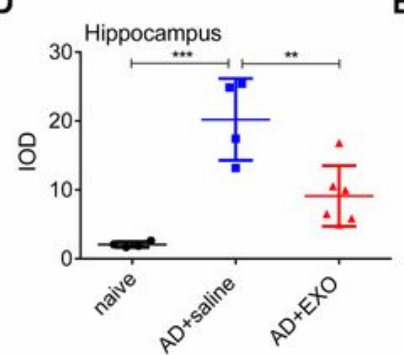

E

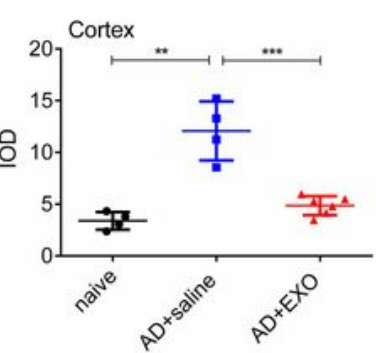

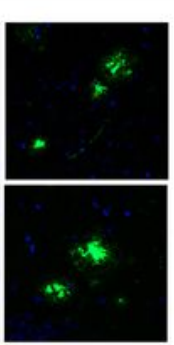

Hippocampus

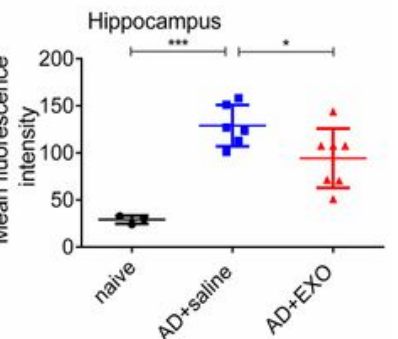

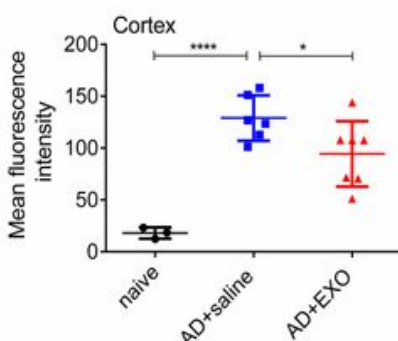

L

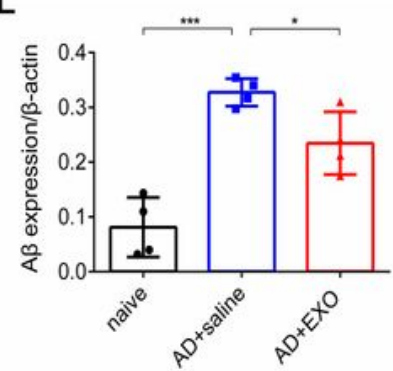

Figure 2

Tail vein injection of MSC-EXO reduces the A $\beta$ aggregation in APP/PS1 mice. (A) A 6E10 immunohistochemistry images present the deposition of $A \beta$ in the hippocampal and cortical regions of experimental mice. Areas in squares, at higher magnification, show the distinction of $A \beta$ aggregation in 
hippocampus $(\mathrm{H})$ and cortex $(\mathrm{C})$ among three groups. IOD and area ratio are used to quantify plaque load $(B-E)$. (F) Thioflavine $S$ staining also present MSC-EXO-mediated changes in terms of A $\beta$ aggregation in hippocampus and cortex, and in the right panel, $A \beta$ deposition (green) is shown at higher magnification. Plaque quantification in hippocampus and cortex appears as area ratio $(G, H)$ and mean fluorescence intensity $(I, J)$. (K) Western blotting of protein expression of $A \beta$. (L) Statistic analysis of relative expression in each group. Quantification data are expressed with mean $\pm S E M$. ${ }^{*} P<0.05,{ }^{\star \star} P<0.01$, $\star \star \star * P<0.001, * \star \star \star P<0.0001$.

A

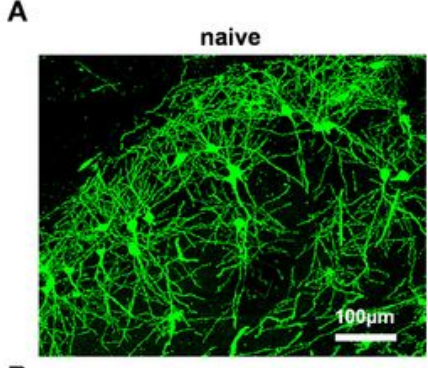

B

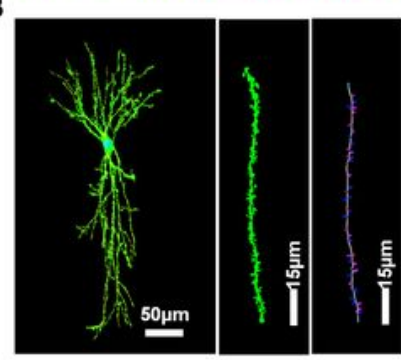

C

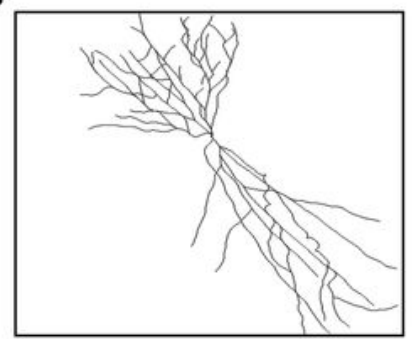

D

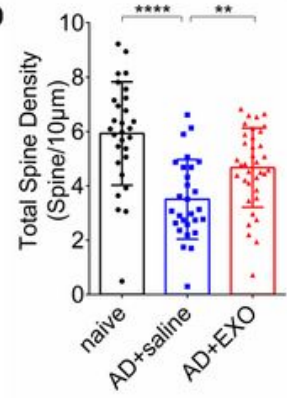

H

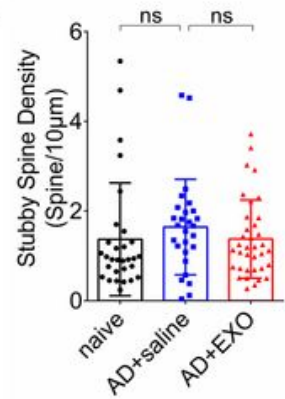

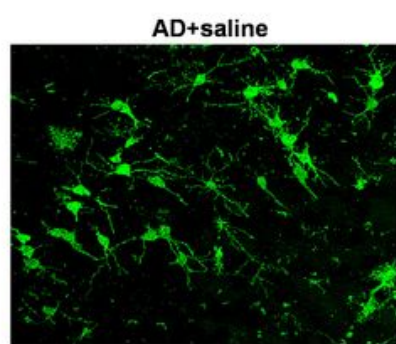
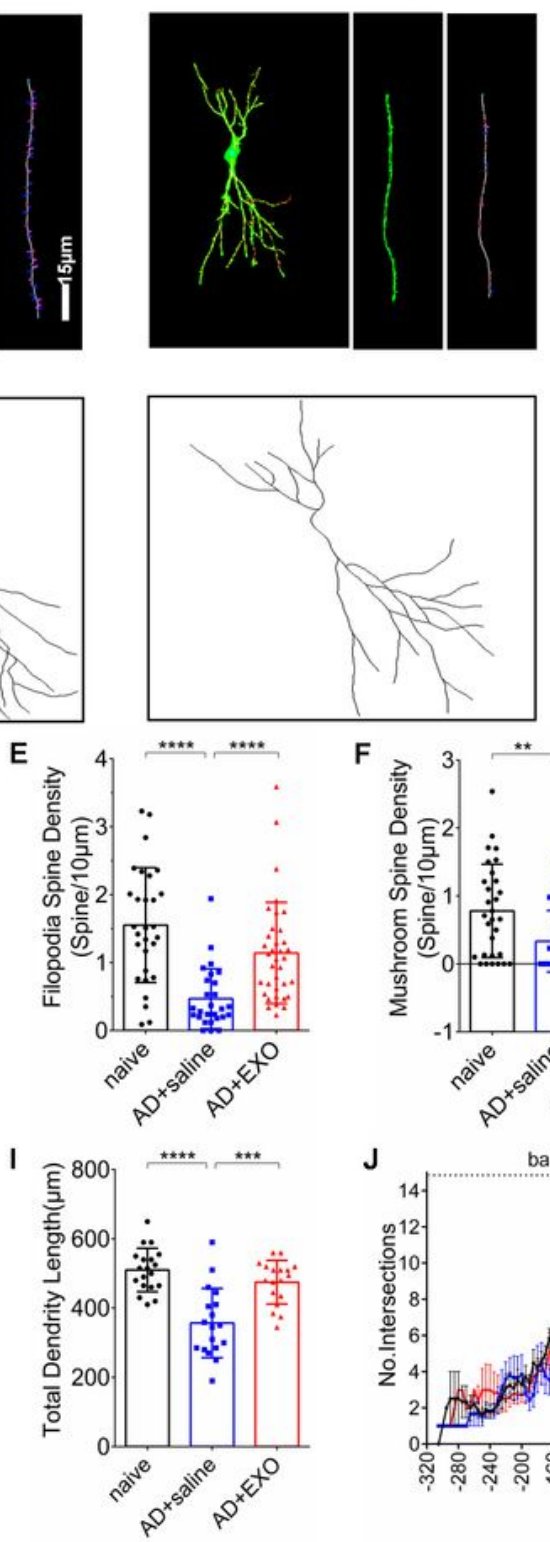
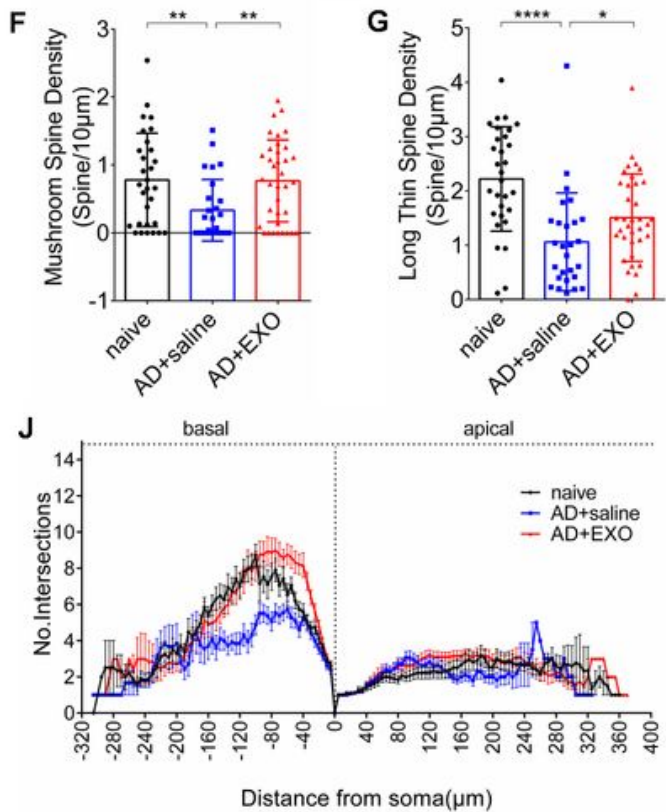

G

- naive AD+EXO

Distance from soma $(\mu \mathrm{m})$
$A D+E X O$
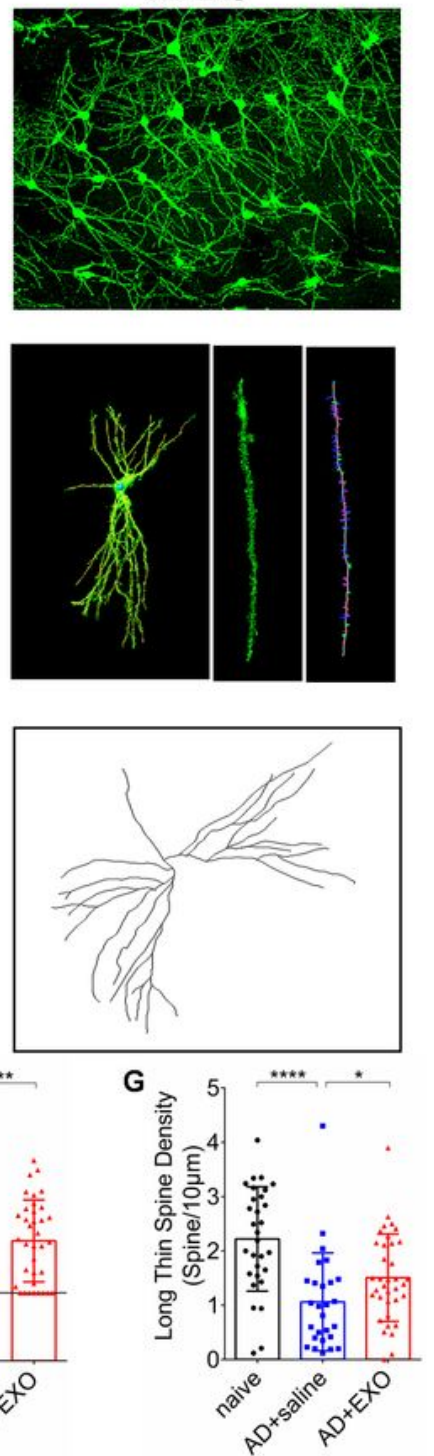


\section{Figure 3}

MSC-EXO administration repairs the hippocampal neuronal morphology alterations in mice. Alterations of synaptic plasticity were examined using Golgi-Cox staining. (A) Representative Golgi-Cox staining images of the neuronal morphology in the CA1 region of each group. (B) 3D reconstruction of spine and dendritic phenotype of hippocampal neurons, and images of classification of dendritic spines. Imaris analysis of spines along basal dendrites including total spine density (D), filopodia spine density (E), mushroom spine density $(F)$, long thin spine density $(G)$ and stubby spine density $(H)$ of three groups. (C) Representative neurite images for Sholl analysis of Golgi images of pyramidal neurons in naive, $A D+$ saline and $A D+E X O$ group. Statistical analysis of total dendritic length (I) and dendritic intersections $(J)$ in the lower panel. Values represent mean \pm SEM except the dendritic intersections as mean \pm SEM. ${ }^{\star} P<0.05$, ${ }^{\star \star} P<0.01$, ${ }^{\star \star *} P<0.001$, ${ }^{\star \star \star *} P<0.0001$. 
A

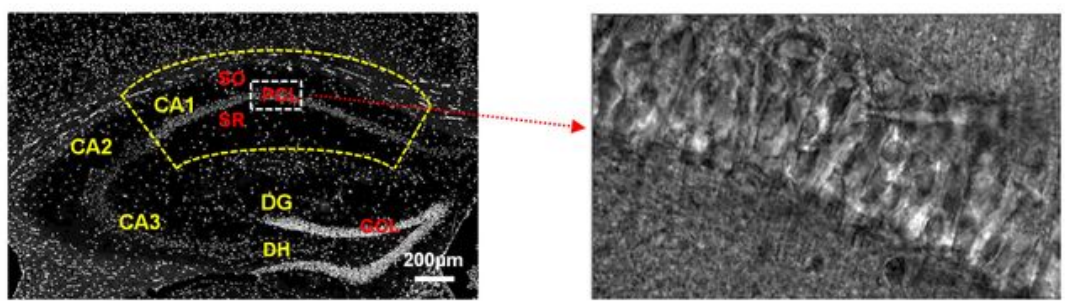

B

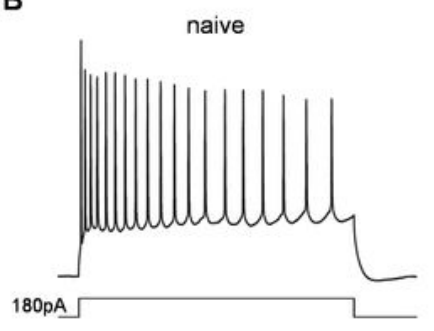

AD+saline

$A D+E X O$

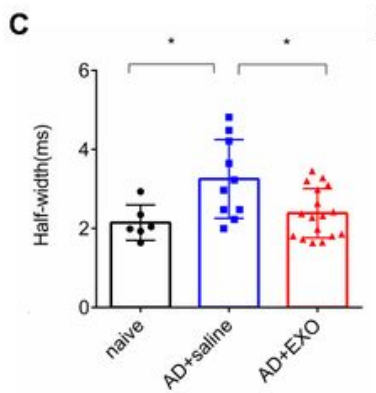

F

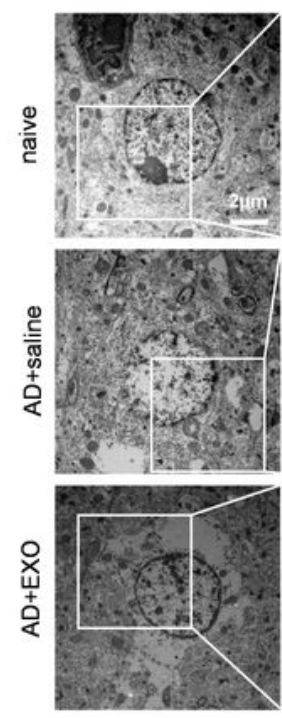

D

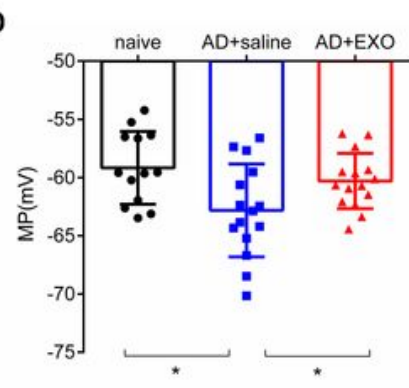

G
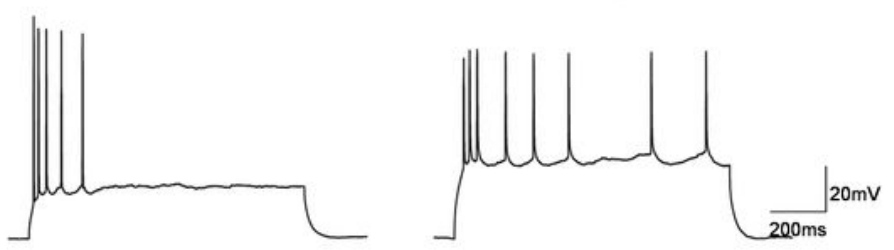

E

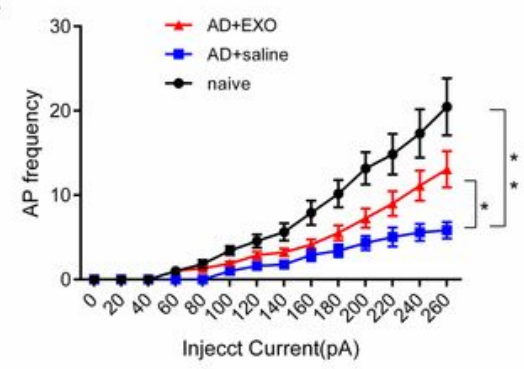

H

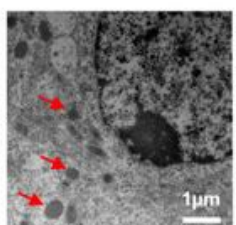

cox IV

том20

FIS1

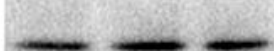

$\beta$-actin
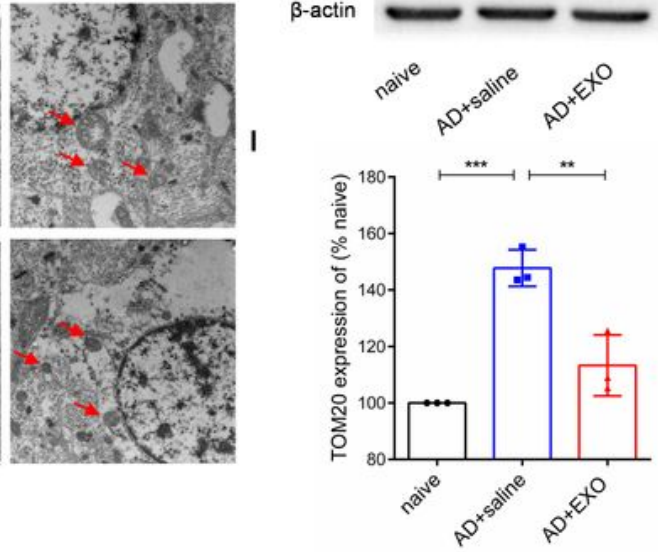

J
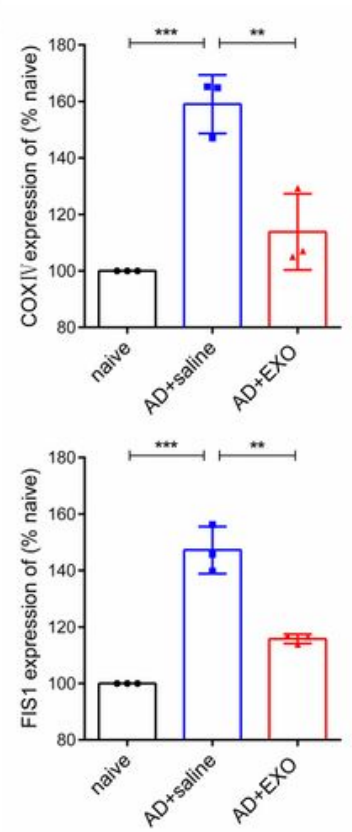

\section{Figure 4}

Exosome therapy restores neuronal excitability and mitochondrial changes in the AD model. (A) Anatomical diagram of the hippocampus, and hippocampal CA1 pyramidal neurons are used for whole cell patch clamp recording. (B) The waveform of the pyramidal neurons in each group. Statistic data of neuronal MP (D), half-width (C) of AP and AP frequency (E) in the naive, AD+saline and AD+EXO groups. (F) Representative TEM scanning images of hippocampal CA1 pyramidal neurons in each group, and on 
the right, higher magnification used to observe mitochondrial changes (arrowhead). (G) Western blotting of the relative expression of COX $\otimes(H), T O M 20(I)$ and FIS1 $(J)$ in the naive, AD+saline and AD+EXO groups. Most values were presented as Mean \pm SEM, other than data of AP frequency as Mean \pm SEM. ${ }^{*} \mathrm{P}<0.05,{ }^{* *} \mathrm{P}<0.01,{ }^{*} * \mathrm{P}<0.001, * * \star * \mathrm{P}<0.0001$.

A

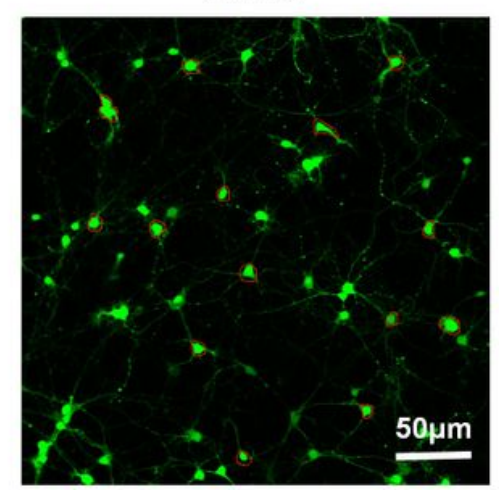

B

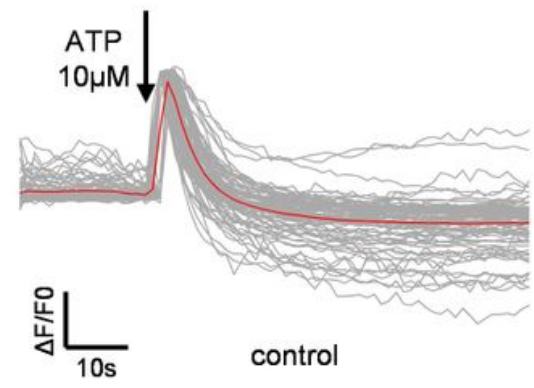

C

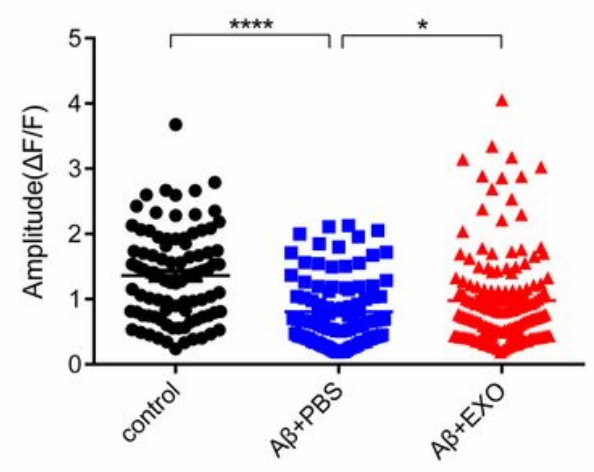

$A \beta+P B S$
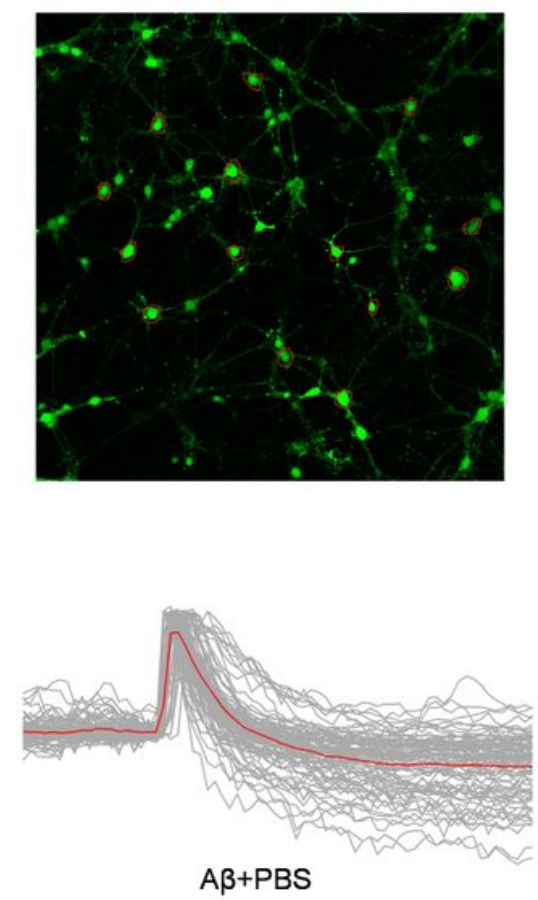

D

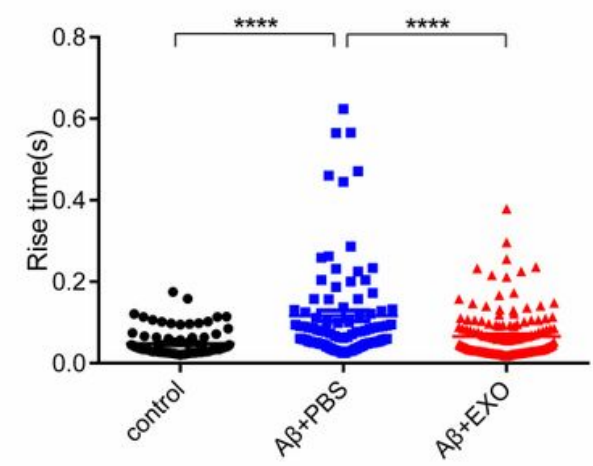

$A \beta+E X O$
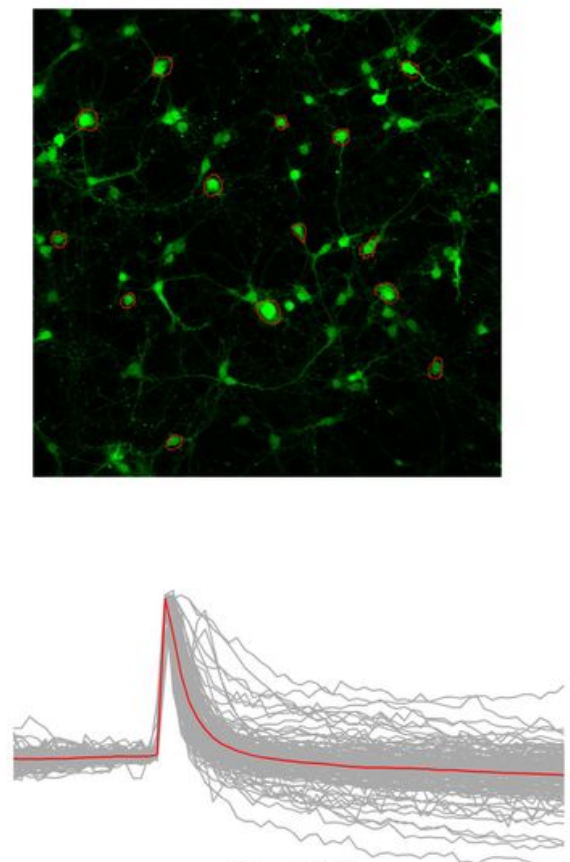

$A \beta+E X O$

E

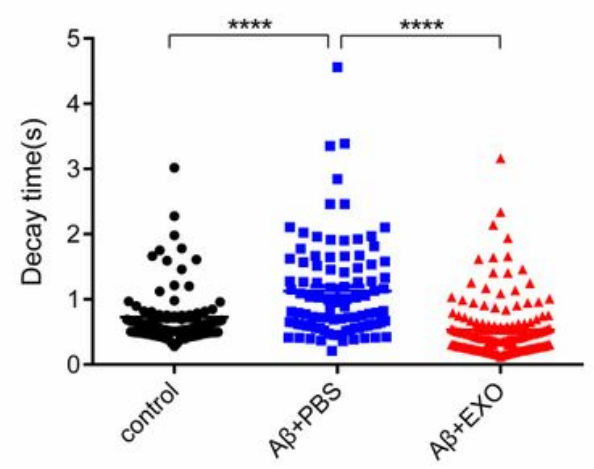

\section{Figure 5}

MSC-EXO treatment ameliorates calcium transients in A -induced primary culture of hippocampal neurons. The intracellular $\mathrm{Ca} 2+$ oscillations were detected to use a calcium indicator Fluo-8 AM in primary hippocampal neurons. (A) Confocal microscope images of $\mathrm{Ca} 2+$ imaging in cultured primary neurons using Fluo-8 AM in control, $A \beta+P B S, A \beta+E X O$ groups (D). (B) Igor software assay shows the two phases (20 and 50 seconds, respectively) of the intracellular Ca2+ oscillations in each group. Statistical analysis data of the amplitude $(\Delta F / F)(C)$, rise time $(D)$, and decay time $(E)$ of the Ca2+ 
signaling after adding ATP in each group are expressed as scatter plots with mean $\pm S D$. ${ }^{*} P<0.05$, $\star * P<0.01, * \star * P<0.001, * * \star * P<0.0001$.

A
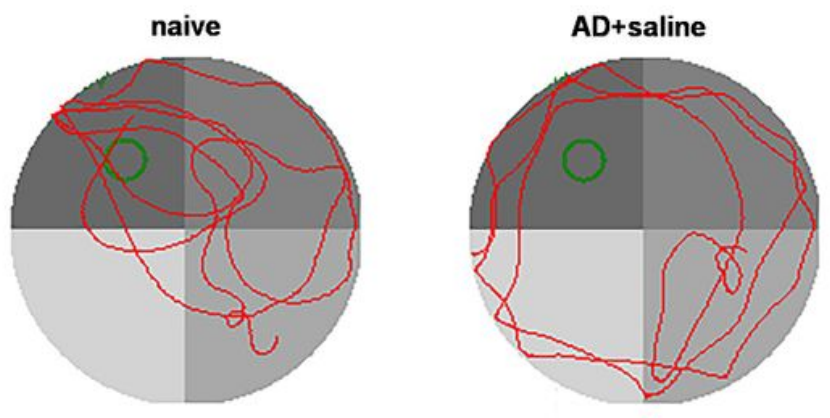

C

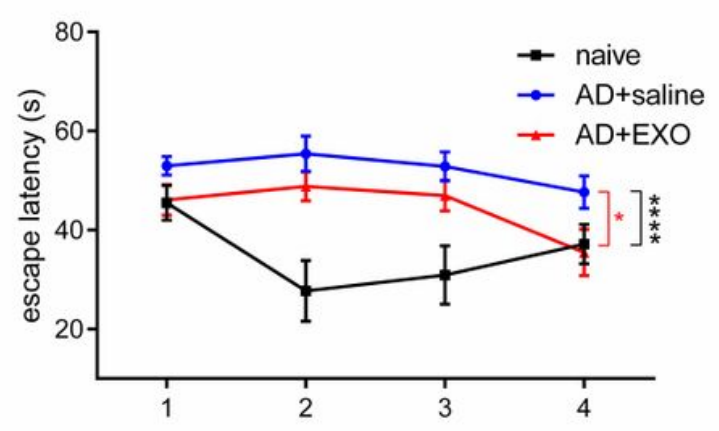

$\mathbf{F}$

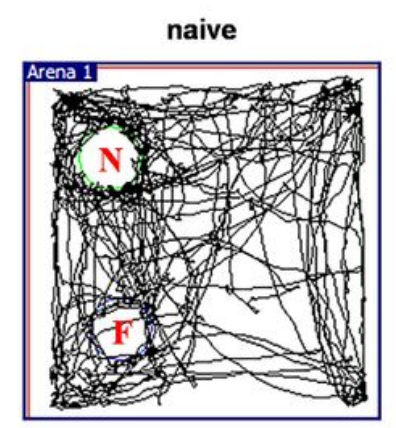

AD+saline

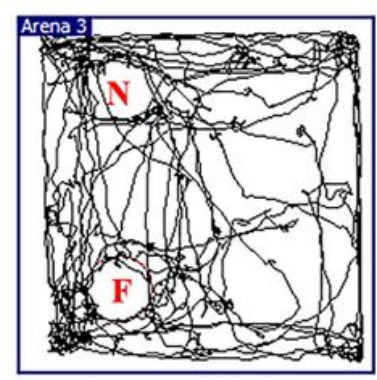

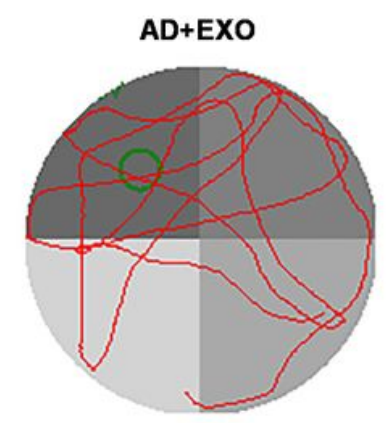

D

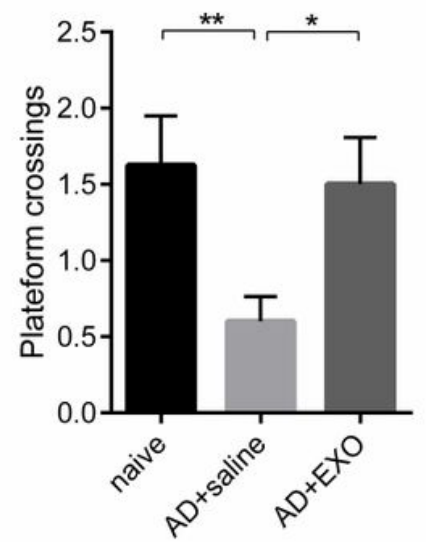

AD+EXO

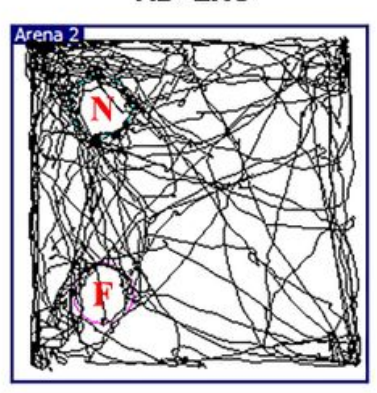

B

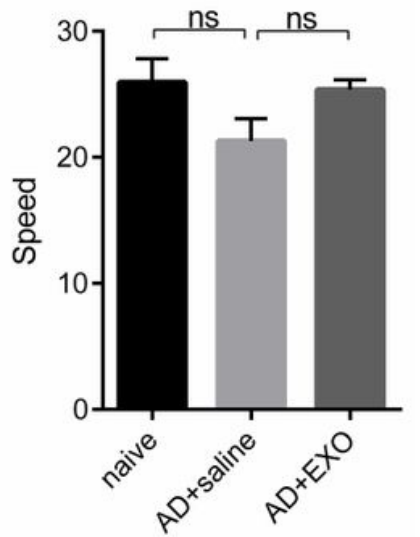

E

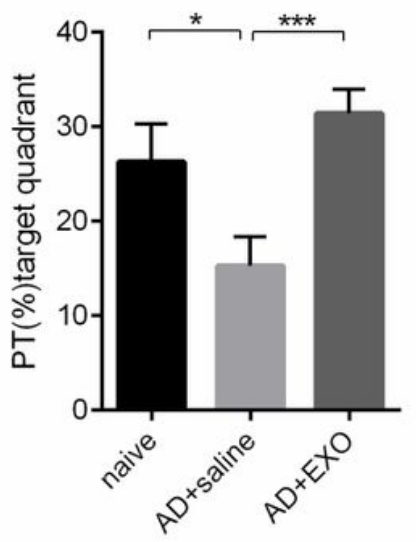

G

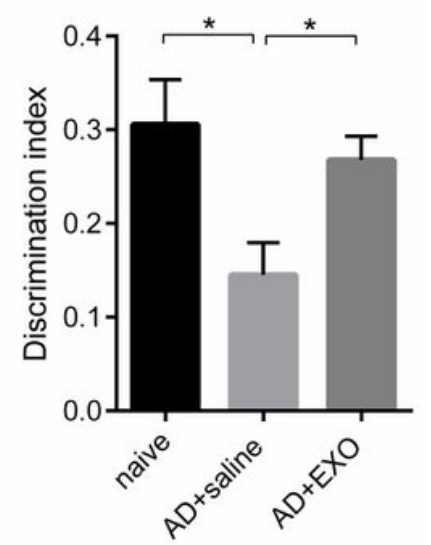

Figure 6

Exosomes treatment improves cognitive deficits in the APP/PS1 mice. MWM and NORT were used to detect learning and memory impairments of mice. $(A)$ The trajectory of three groups mice in MWM test. (C) In the spatial learning session, escape latency of each group was analyzed. And in the probe test, speed $(\mathrm{mm} / \mathrm{s})(B)$, platform crossings (D) and the PT (\%)-target quadrant (E) in different experimental groups were analyzed. (F) Motion trail of each group in NORT (novel object, N; familiar object, F). (G) 
Comparison of the discrimination index of naive, $A D+$ saline and $A D+E X O$ groups mice. All values represent mean \pm SEM. ${ }^{*} \mathrm{P}<0.05,{ }^{*} \mathrm{P}<0.01,{ }^{*} * * \mathrm{P}<0.001$.

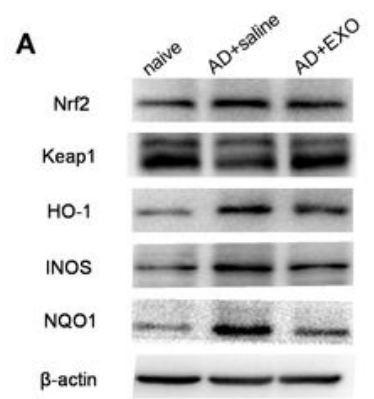

D
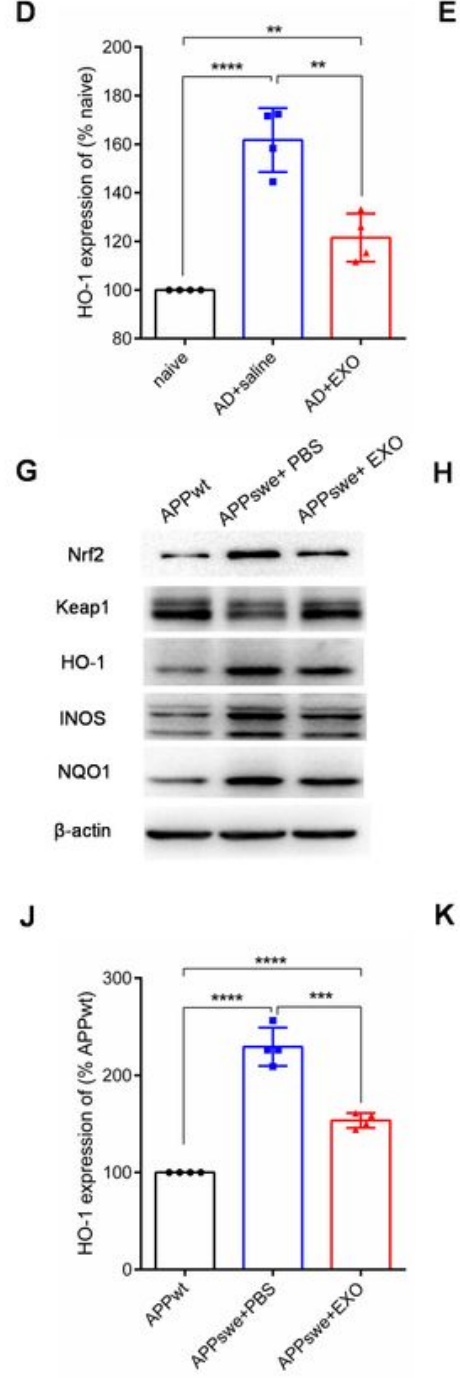

B

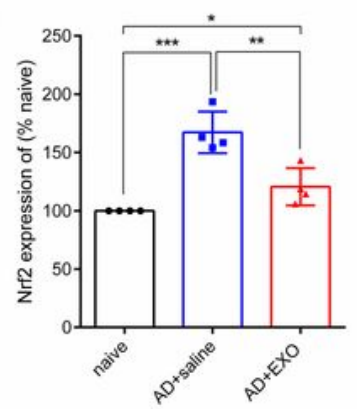

E

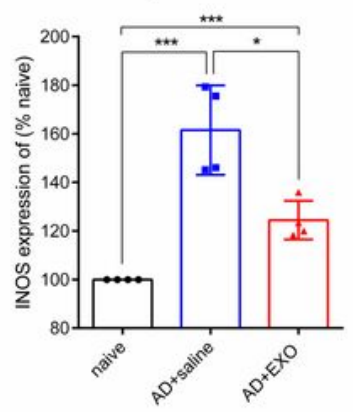

$\mathrm{H}$

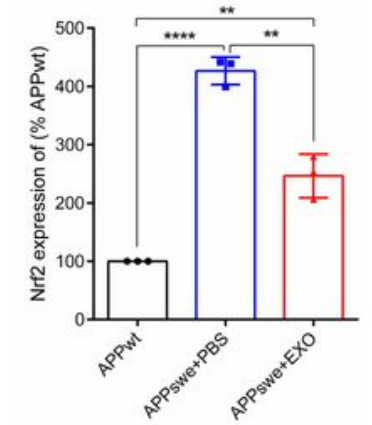

K

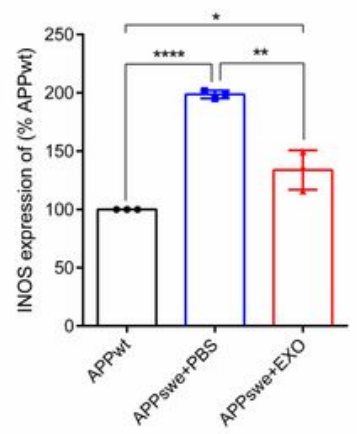

C

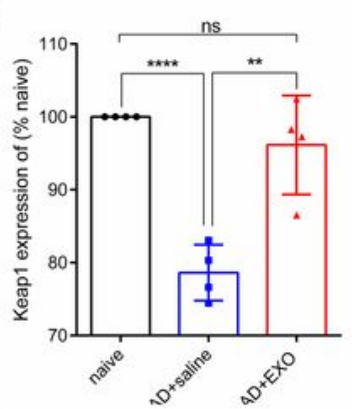

$\mathbf{F}$

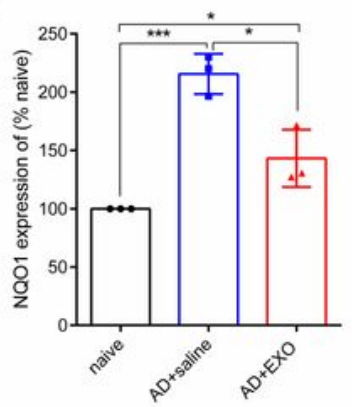

I

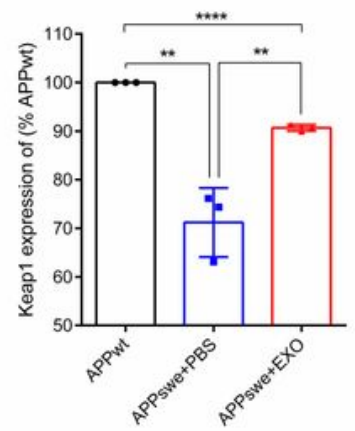

L

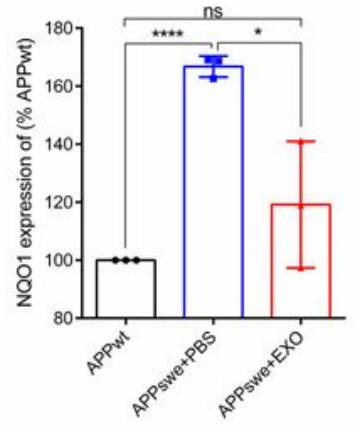

Figure 7

Oxidative defense system is associated with the exosome therapy in APP/PS1 mice. Western blotting was used to detect Nrf2 signaling in vivo and vitro. (A) Western blotting of the relative expression of Nrf2 (B), Keap1 (C), HO-1 (D), INOS (E) and NQ01 (F) in the naive, AD+saline and AD+EXO groups mice. (G) 
Representative image of Western blotting of Nrf2 signaling in APPwt, APPswe + PBS and APPswe + EXO groups. Statistical analysis of the relative expression of Nrf2 (H), Keap1 (I), HO-1 (J), INOS (K) and NQ01 $(\mathrm{L})$ in vitro. Statistical difference between groups is shown in the histograms. Values represent mean \pm SEM. ${ }^{*} P<0.05,{ }^{\star *} P<0.01,{ }^{*} * * P<0.001,{ }^{*} * \star * P<0.0001$.

\section{Supplementary Files}

This is a list of supplementary files associated with this preprint. Click to download.

- GraphicalAbstract.jpg 